\title{
A Comparative Study of Nonsingular Terminal Sliding Mode and Backstepping Schemes for the Coupled Two-Tank System
}

\author{
Safa Choueikh, ${ }^{1}$ Marwen Kermani $\mathbb{D}^{1,2}$ and Faouzi M'sahli ${ }^{1}$ \\ ${ }^{1}$ Laboratory of Automation, Electrical Systems, and Environment, National Engineering School of Monastir, \\ Ibn El Jazzar, Skaness 5019, Monastir, University of Monastir, Tunisia \\ ${ }^{2}$ National School of Advanced Science and Technology of Borj Cedria, BP-122 Hammam-Chott 1164, University of Carthage, \\ Carthage, Tunisia \\ Correspondence should be addressed to Marwen Kermani; kermanimarwen@gmail.com
}

Received 8 January 2021; Revised 7 March 2021; Accepted 6 October 2021; Published 3 November 2021

Academic Editor: Weixiang Zhou

Copyright (C) 2021 Safa Choueikh et al. This is an open access article distributed under the Creative Commons Attribution License, which permits unrestricted use, distribution, and reproduction in any medium, provided the original work is properly cited.

\begin{abstract}
This paper presents an implementation of two radically different control schemes for a state-coupled two-tank liquid-level system. This is due to the purpose of transferring theoretical studies to industrial systems. The proposed schemes to be introduced and compared are the nonsingular terminal sliding mode control (NTSMC) and the backstepping control (BC). The performances of the developed methods are experimentally tested on a particular class of second-order nonlinear systems. The main purpose of the considered control schemes is to achieve a tracking trajectory for a coupled-tank system. It is proved that the designed robust controllers guarantee the stability of the corresponding closed loop systems. The obtained results are verified with the same setup test to ensure a suitable basis for their comparison. During the experiments, we resorted to adding an integrator to the backstepping control so that we improve the results, leading to the appearance of the integrator backstepping control (IBC). To focus on the adequacy and applicability of the suggested control layout, theoretical comparisons as well as experimental results are afforded and debated.
\end{abstract}

\section{Introduction}

Liquid-level control systems can be classified as an important process not only for draining but also in several major industries [1]. Various systems and devices have been developed to control the liquid level such as in food processing, water purification systems, filtration, pharmaceutical industries, decoration, boilers, beverage, and industrial chemical processing. Liquid level is so important and there are many parameters that should be mastered to measure it.

In the few past decades, some researchers have invented the design and the implementation of the liquid level of a coupled-tank system controller such as the ProportionalIntegral-Derivative (PID) type controllers [2], the backstepping controller $[3,4]$, the nonlinear constrained predictive algorithms based on the feedback linearization control [5], the second-order sliding mode control [6], Constrained Pole Assignment Control (CPAC) [7, 8], and neurofuzzy sliding mode controller (NFSMC) [9]. Therefore, industrial process control engineering has immensely benefited from the technology development brought by digital computers and their sophisticated software. Thus, these advanced technologies have allowed the ability of implementing advanced control algorithms that have been considered as quite complex in their implementation. In addition, the industry demands as well as the high precision required by modern systems encourage research in control engineering to develop and synthesize robust nonlinear control algorithms.

Up to now, among the satisfactory controls which prove a good robustness against uncertainties and allow disturbance rejections, we mention the linearization control (LC) $[10,11]$, the robust control (RC) [12], the model predictive control (MPC) $[13,14]$, the backstepping control (BC) $[15,16]$, and the sliding mode control (SMC) [17]. The MPC and the $\mathrm{BC}$ have especially been addressed to design robust 
controllers in many physical systems. For example, the $\mathrm{BC}$ has proven its effectiveness in several applications such as two-tank system [3], spacecraft [18], and quadrotor unmanned aerial vehicles [19]. The SMC has been applied on several practical systems such as underactuated ships [20], flexible-link manipulator [21], gearless PMSG-based wind turbine [22], magnetic levitation [23], underwater vehicles [24], DC motor-controlled inverted pendulum [25], and autonomous airship [16].

Despite practical control processes have been solved based on the above-mentioned approaches, these techniques require a measured state vector and a precise model.

As it is well known, the SMC is an efficient robust control for uncertain systems [26, 27] and bounded external disturbances [27]. This approach consists of two steps. First, the system state path reaches a predefined surface according to the control objectives, called sliding surface. Then, the designed control restricts the system trajectory to remain on this surface and to converge into its equilibrium state. Indeed, this task has been achieved by introducing a discontinuous term allowing a high frequency switching of system trajectories around the sliding surface. However, this procedure generally leads to the flawed "chattering phenomenon" which may generate some serious problems during the experimental applications. Some attention has been drawn to reducing the effect of this chattering phenomenon, which has led to other new scheme strategies based on the principle of SMC.

Among these scheme strategies, we can mention the terminal sliding mode control (TSMC) [28-30], the fast terminal sliding mode Control (FTSMC) [31], the integral terminal sliding mode control (ITSMC) [32, 33], the nonsingular terminal sliding mode control (NTSMC) [34, 35], and the fast nonsingular integral terminal sliding mode control (FNITSMC) [36]. In this framework, the TSMC has been designed to achieve the finite-time convergence of the system dynamics and it has been applied in many practical processes such as the rigid robotic manipulators $[37,38]$, the PWM-based DC-DC [39], and robotic airships [40].

Compared to the conventional SMC, the TSMC which has a nonlinear sliding surface offers superior properties such as speed, convergence in finite time, and more accurate control [28]. However, it has two inconveniences which are, respectively, the singularity point problem and the requirement of the uncertainty limit problem. The first one can be overcome by the nonsingular approach in the NTSMC, and the second one can be solved through a well-designed uncertainty estimation [28, 29].

To the best of authors' knowledge, there is no result in the literature applying TSMC to the two-tank system which motivates us to present this study.

This paper investigates a comparative study between NTSMC, BC, and IBC for the two-tank liquid-level system which has not been discussed yet. Indeed, more suitable control approaches have been considered to guarantee the desired performance for the liquid-level system.

The main contributions of this paper are summarized as follows:
The NTSMC, BC, and IB can be suggested among the most widespread and well-performing control approaches which have been simulated and implemented for a coupled two-tank system.

The closed loop stability proofs of the NTSMC and BC schemes in the sense of Lyapunov have been developed.

A theoretical and practical comparison for the interest of practicing engineers and researchers has been addressed.

This paper is structured as follows: In Section 2, the statecoupled two-tank system description and its mathematical model will be introduced. The NTSMC liquid-level approach to be considered is presented and its stability is studied in Section 3. Section 4 introduces the model based backstepping liquid-level controller and the study of its stability. A comparison of the control approaches, based on the proposed theoretical analysis, is presented in Section 5. Section 6 introduces the simulation and experimental results. The conclusion and the future works are given in Section 7 .

\section{System Description}

The model of a two degrees of freedom (DOF) state-coupled two-tank system is given in Figure 1. This system is composed of a liquid basin, a pump, and two equal-volumed tanks. These tanks are equipped with "in" and "out" orifices and level sensors at their bottoms.

This system works as follows: First, the pump absorbs liquid from the basin to discharge it into tank 2. After that, the liquid exits from tank 2 to fill in tank 1 . Finally, tank 1 liquid is emptied into the basin.

The main characteristics and constraints of the dynamic system model are given as follows:

The system input is the voltage $u$ delivered by the pump which varies between $0 \mathrm{~V}$ and $12 \mathrm{~V}$ while the system output is the liquid level $h_{1}$

A pressure sensor is associated with both tank 1 and tank 2 for instantaneous measurement of the levels $h_{1}$ and $h_{2}$

The liquid levels $h_{1}$ and $h_{2}$ belong to the interval $[4 \mathrm{~cm}, 30 \mathrm{~cm}$ ]

According to the already mentioned description, the dynamic equations of the liquid level in the two tanks are obtained as follows: by

The time change rate of liquid level in each tank is given

$$
\dot{h}_{i}(t)=\frac{1}{S_{i}}\left(F_{i}^{\text {in }}(t)-F_{i}^{\text {out }}(t)\right), \quad i=1,2,
$$

where $h_{i}(t), S_{i}, F_{i}^{\text {in }}(t)$, and $F_{i}^{\text {out }}(t)$ are the liquid level, crosssectional area, and inflow and outflow rates, respectively, for the $i^{\text {th }}$ tank. Next, the inflow rate into tank 2 is given by

$$
F_{2}^{\text {in }}(t)=K_{p} u(t),
$$




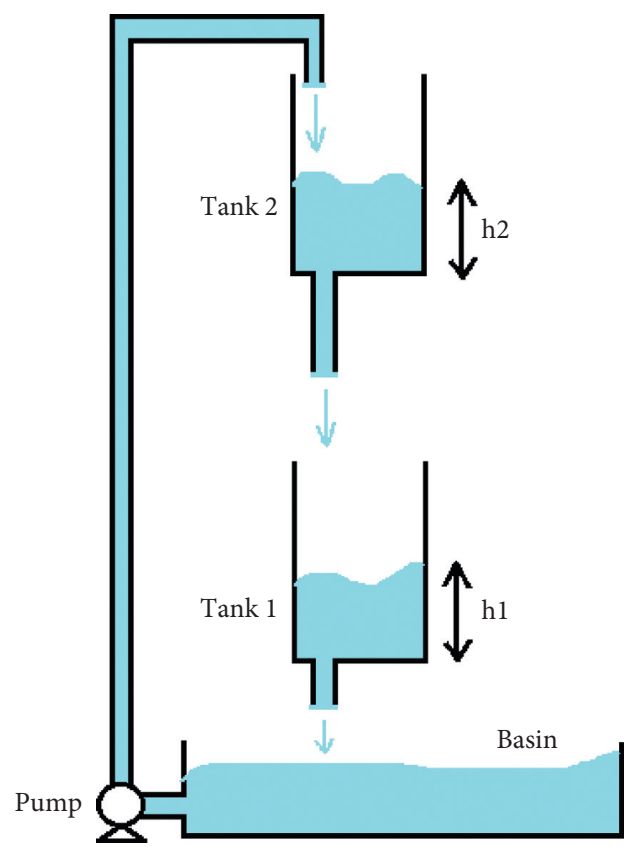

FIGURE 1: State-coupled two-tank system.

where $K_{p}$ is the pump constant $\left(\mathrm{cm}^{3} / \mathrm{V}\right)$ and $u(t)$ is the voltage applied to the pump, such that, using Bernoulli's law for the flow through small orifices, the outflow velocity from the orifice at the bottom of each tank is

$$
v_{i}^{\text {out }}(t)=\sqrt{2 g h_{i}(t)}, \quad i=1,2 .
$$

Then, the outflow rate for each tank is given in

$$
F_{i}^{\text {out }}(t)=s_{i} \sqrt{2 g h_{i}(t)}, \quad i=1,2,
$$

where $g$ is the gravitational acceleration and $s_{i}$ denotes the cross-sectional area of the outflow orifice at the bottom of the $i^{\text {th }}$ tank.

Finally, we note that for the two-tank liquid-level system:

$$
F_{i}^{\text {in }}(t)=F_{2}^{\text {out }}(t)
$$

Thus, we obtain the following dynamic equations of the system:

$$
\left\{\begin{array}{l}
\dot{h}_{1}(t)=-\frac{s_{1}}{S_{1}} \sqrt{2 g h_{1}(t)}+\frac{s_{2}}{S_{1}} \sqrt{2 g h_{2}(t)}, \\
\dot{h}_{2}(t)=-\frac{s_{2}}{S_{2}} \sqrt{2 g h_{2}(t)}+\frac{K_{p}}{S_{2}} u(t) .
\end{array}\right.
$$

For convenience, in stating the main result of this section, we define the following constants: $c_{2}=\left(s_{1} / S_{1}\right) \sqrt{2 g}, c_{1}=\left(K_{p} / S_{2}\right), \quad a=\left(s_{2} / S_{1}\right) \sqrt{2 g}, b=\left(s_{2} / S_{2}\right)$ $\sqrt{2 g}$.

Consider that there is the same cross-sectional area for the two tanks: $S_{1}=S_{2}$.

In such conditions, (6) can be written as the following system:

$$
\left\{\begin{array}{l}
\dot{h}_{1}(t)=-c_{2} \sqrt{h_{1}(t)}+c \sqrt{h_{2}(t)} \\
\dot{h}_{2}(t)=-c \sqrt{h_{2}(t)}+c_{1} u(t) \\
y=h_{1}(t) .
\end{array}\right.
$$

In what follows, we assume that the two sensors are available to measure the liquid levels in the two tanks. The level of $h_{1}$ will be controlled to follow a reference trajectory.

\section{Nonsingular Terminal Sliding Mode Control}

3.1. Typical Terminal Sliding Mode Control. To summarize the basic principle of TSMC, we consider the following second-order system [28]:

$$
\left\{\begin{array}{l}
\dot{x}_{1}=x_{2} \\
\dot{x}_{2}=f(x)+g(x) u+d(x, t)
\end{array}\right.
$$

where $x_{1}$ and $x_{2}$ are the system states, $f(x)$ and $g(x) \neq 0$ are the known nonlinear functions, respectively, $u$ is the control input, and $d(x, t)$ is the disturbance such as $|d(x, t)| \leq L$ where $L>0$.

The sliding variable is selected as

$$
S=x_{2}+\frac{1}{\beta} x_{1}^{q / p},
$$

where $\beta>0, p, q$, and $(p>q)$ are positive odd numbers.

The controller is designed as

$$
u=-g^{-1}(x)\left(f(x)+\beta \frac{q}{p} x_{1}^{(q / p)-1} x_{2}+(L+\eta) \operatorname{sgn}(S)\right) .
$$

From (10), we have $(q / p)-1<0$. When $x_{1}=0$ and $x_{2} \neq 0$ a singularity problem exists for the typical terminal controller. Thus, a nonsingular terminal sliding mode control method is proposed by [31] to deal with the singularity problem.

The nonsingular sliding variable is designed as

$$
S=x_{1}+\frac{1}{\beta} x_{2}^{p / q},
$$

where $\beta>0, p, q$, and $p>q$ are positive odd numbers.

The nonsingular sliding mode controller is given as

$$
u=-g^{-1}(x)\left(f(x)+\beta \frac{q}{p} x_{2}^{2-(p / q)}+(L+\eta) \operatorname{sgn}(S)\right),
$$

where $1<(p / q)<2$ and $\eta>0$.

In the next section, scheme (12) will be developed to deal with tracking problem position.

3.2. Nonsingular Terminal Sliding Mode Control for the Coupled Two-Tank System. Many methods have been suggested to avoid the singularity problem in the typical TSMC. The first approach to be mentioned switches the sliding mode from TSMC to linear hyperplane-based sliding mode [28]. The second approach transfers the trajectory to a predefined open region where TSMC is not singular [29]. 
These methods advocate indirect approaches to avoid singularity. In this work, a simple NTSMC, which is completely capable of overcoming this problem, is highly recommended. The suggested NTSMC model is interpreted as follows:

The first step consists of transforming the model of the real system into an affine control model, described in (8). Starting from the model given in (7), we consider the following diffeomorphism:

$$
x=\left[\begin{array}{l}
x_{1} \\
x_{2}
\end{array}\right]=\vartheta(h)=\left[\begin{array}{l}
h_{1} \\
\dot{h}_{1}
\end{array}\right]=\left[\begin{array}{c}
h_{1} \\
-c_{2} \sqrt{h_{1}}+c \sqrt{h_{2}}
\end{array}\right] .
$$

The time derivative of the new coordinates gives

$$
\left\{\begin{array}{l}
\dot{x}_{1}=\dot{h}_{1}, \\
\dot{x}_{2}=\frac{d\left(-c_{2} \sqrt{h_{1}}+c \sqrt{h_{2}}\right)}{d t}=-\frac{c_{2}}{2}\left(\frac{\dot{h}_{1}}{\sqrt{h_{1}}}\right)+\frac{c}{2}\left(\frac{\dot{h}_{2}}{\sqrt{h_{2}}}\right), \\
y=x_{1}(t) .
\end{array}\right.
$$

Since we have $\dot{h}_{2}=-c \sqrt{h_{2}}+c_{1} u$ and $\sqrt{h_{2}}=\left(x_{2}+c_{2} \sqrt{x_{1}}\right) / c$, we obtain

$$
\left\{\begin{array}{l}
\dot{x}_{1}=x_{2} \\
\dot{x}_{2}=f(x)+g(x) u \\
y(t)=x_{1}(t)
\end{array}\right.
$$

where $\quad f(x)=-\left(c_{2} / 2\right)\left(x_{2} / 2 \sqrt{x_{1}}\right)-\left(c^{2} / 2\right) \quad$ and $g(x)=\left(c^{2} c_{1} / 2\left(x_{2}+c_{2} \sqrt{x_{1}}\right)\right)$.

In the sequel, we apply the inverse of the diffeomorphism $\vartheta^{-1}$ to recuperate the state in $h$ for the controller.

The inverse of the diffeomorphism is as follows:

$$
h=\vartheta^{-1}(x)=\left[\begin{array}{l}
h_{1} \\
h_{2}
\end{array}\right]=\left[\begin{array}{c}
x_{1} \\
\frac{x_{2}+c_{2} \sqrt{x_{1}}}{c}
\end{array}\right] .
$$

Figure 2 illustrates the proposed NTSM controller as follows.

The nonsingular adaptive sliding variable is designed by

$$
S=e_{1}+\frac{1}{\beta} e_{2}^{p / q}
$$

with

$$
\left\{\begin{array}{l}
e_{1}=x_{1}-r \\
e_{2}=x_{2}-\dot{r}
\end{array}\right.
$$

where $x_{1}, x_{2}$ are states of the system and $r$ is a desired trajectory. $\beta>0, p, q, p>q$, and $1<(p / q)<2$ are positive odd numbers.

3.3. Stability Analysis of the NTSM Controller Design. In what follows, the stability analysis of the corresponding closed loop system deduced from (15) will be addressed by using the NTSMC. We extend scheme (12) to overcome the tracking problem position. For this, we propose Theorem 1 as follows.

Theorem 1. For system (13), the NTSMC law is designed as follows:

$$
u=-g^{-1}(x)\left(f(x)-\ddot{r}+\beta \frac{q}{p} e_{2}^{(2-(p / q))}+(L+\mu) \operatorname{sign}(S)\right),
$$

where (19) will be reached in finite time and the errors $e_{1}$ and $e_{2}$ will converge to zero in finite time if there exist real parameters $\mu, L, p, q$, and $\beta$ such as $\mu>0, L>0$, and $1<(p / q)<2$.

Proof. Consider the following Lyapunov function for the resulting closed loop system inspired from (20) and (21):

$$
V=\frac{1}{2} S^{2}
$$

Its derivative along the closed loop system given in (13) and (15) is deduced as

$$
\dot{V}=S \dot{S},
$$

where

$$
\dot{S}=\left(\dot{x}_{1}-\dot{r}\right)+\frac{1}{\beta} \frac{p}{q}\left(\dot{x}_{2}-\ddot{r}\right)\left(x_{2}-\dot{r}\right)^{((p / q)-1)} .
$$

From (22) and (15), we deduce

$$
\dot{S}=\left(x_{2}-\dot{r}\right)+\frac{1}{\beta} \frac{p}{q}\left(x_{2}-\dot{r}\right)^{((p / q)-1)}\left(-\frac{c_{2}}{2} \frac{x_{2}}{\sqrt{x_{1}}}-\frac{c^{2}}{2}+\frac{c^{2} c_{1}}{2\left(x_{2}+c_{2} \sqrt{x_{1}}\right)} u-\ddot{r}\right) .
$$

According to (19), we have

$$
\dot{S}=\left(x_{2}-\dot{r}\right)+\frac{1}{\beta} \frac{p}{q}\left(x_{2}-\dot{r}\right)^{((p / q)-1)}\left(-\beta \frac{q}{p}\left(x_{2}-\dot{r}\right)^{(2-(p / q))}-(L+\eta) \operatorname{sign}(S)\right) .
$$




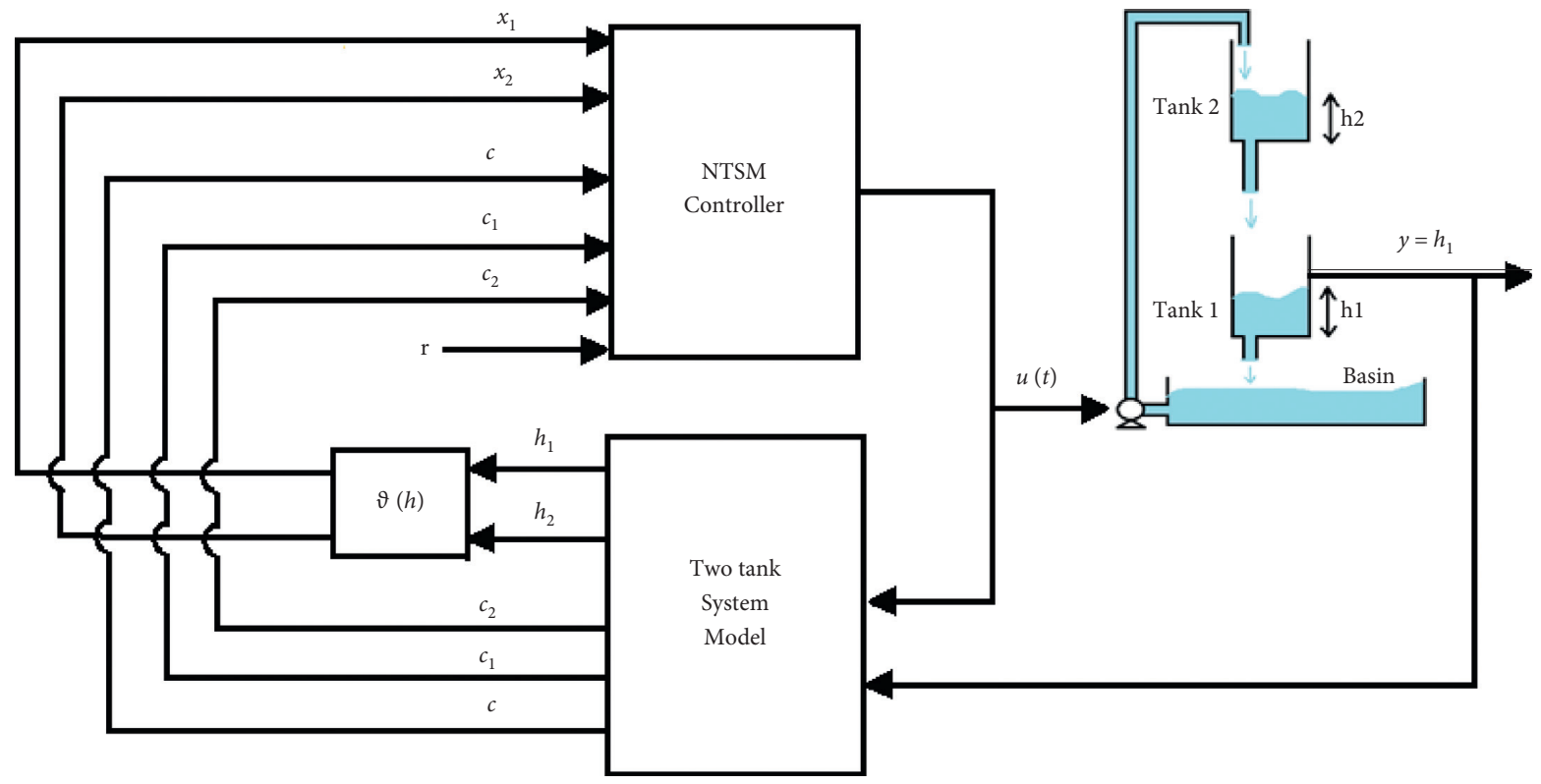

FIgURE 2: Structure of the NTSM scheme.

Finally, we obtain

$$
S \dot{S}=-\frac{1}{\beta} \frac{p}{q}\left(x_{2}-\dot{r}\right)^{((p / q)-1)}(L+\eta) S \operatorname{sign}(S) .
$$

To complete this proof, it should be noticed that NTSMC law (19) is always nonsingular in the state space since $1<(p / q)<2$. Therefore, we assumed that $0<(p / q)-1<1$.

In such condition, we guarantee that $\left(x_{2}-\dot{r}\right) \neq 0$ and $\left(x_{2}-\dot{r}\right)>0$.

Therefore, we can write

$$
\begin{aligned}
& \dot{V} \leq \frac{1}{\beta} \frac{p}{q}\left(x_{2}-\dot{r}\right)^{((p / q)-1)}(-\eta|S|), \\
& \dot{V} \leq-\eta^{\prime}|S|,
\end{aligned}
$$

with $\eta^{\prime}=(1 / \beta)(p / q)\left(x_{2}-\dot{r}\right)^{((p / q)-1)} \eta>0$.

Thus, the Lyapunov stability of the considered system is checked.

This is the end of the proof of Theorem 1.

Remark 1. The proposed NTSMC law in Theorem 1 solves the problem of the control of liquid-level system that represents a special class of systems $(n=2)$. The method proposed can be extended to a class of $n$-order $(n>2)$ nonlinear dynamic systems that represent a broader class of problems:

$$
\left\{\begin{array}{l}
\dot{x}_{1}=f_{1}\left(x_{1}, x_{2}\right), \\
\dot{x}_{2}=f_{2}\left(x_{1}, x_{2}\right)+g\left(x_{1}, x_{2}\right) u,
\end{array}\right.
$$

where $x_{1}=\left(x_{11}, x_{12}, \ldots, x_{1 n}\right)^{T} \in R^{n}, x_{2}=\left(x_{21}, x_{22}, \ldots\right.$, $\left.x_{2 n}\right)^{T} \in R^{n}, f_{1}$ and $f_{2}$ are smooth vector functions, $g$ is a nonsingular matrix, and $u=\left(u_{1}, u_{2}, \ldots, u_{n}\right)^{T} \in R^{n}$ is the control vector.

In fact, this considered approach can be applied to any system, which can be transformed to (27).

\section{Backstepping Control}

One of the benefits of the BC consists in stabilizing the nonlinear system without linearization. In fact, the presence of nonlinearization leads to multiple potential advantages. Therefore, less effort is necessary for the system control. In addition, the corresponding scheme may depend on a less precise information model, which improves the robustness against the modelization errors and assures the global stability.

The $\mathrm{BC}$ aims to use the state as a virtual control. However, the system is then divided into united subsystems in a decreasing order. Thus, the corresponding scheme appears in the last step of the backstepping algorithm. During the stages intermediates, the instability of the nonlinear system is treated, and the order of the system is increased from one step to another. Global stability is guaranteed that it ensures continuity and regulation of nonlinear systems.

4.1. Backstepping Control for the Two-Tank System. We consider the following class of triangular nonlinear SISO systems given in

$$
\left\{\begin{array}{l}
\dot{x}_{1}=\varphi\left(x_{1}\right)+x_{2}, \\
\dot{x}_{2}=v, \\
y=x_{1},
\end{array}\right.
$$

where $y \in R, v \in R,\left(x_{1}, x_{2}\right) \in R^{2}$, and $\varphi\left(x_{1}\right)$ is the nonlinear function.

This considered control aims to stabilize system (28) with the reference trajectory $r \in R$.

In this work, according to the theoretical results in [41], a backstepping controller will be synthesized to ensure the tracking problem for the nonlinear system (28). In addition, the Lyapunov theory has been used to prove the existence of 
some conditions for the chosen feedback gains to guarantee the asymptotic stability of control system (28).

The first step consists in rewriting the two-tank system model (7) in the triangular form as follows:

$$
\left\{\begin{array}{l}
\dot{h}_{1}=-c_{2} \sqrt{h_{1}}+\eta \\
\dot{\eta}=c \frac{\dot{h}_{2}}{2 \sqrt{h_{2}}}=-\frac{c^{2}}{2}+\frac{c c_{1}}{2 \sqrt{h_{2}}} u=v, \\
y=h_{1}
\end{array}\right.
$$

with $\eta=c \sqrt{h_{2}}$.

4.2. Stability Analysis of the Controlled System. The proposed backstepping controller allows guaranteeing the global asymptotic stability of the system output respective to a variable reference signal. This controller is formulated in Theorem 2 as follows.

Theorem 2. We consider system (28) and let $r$ be a reference signal with a bounded derivative and the following assumptions hold:

$$
\begin{aligned}
& A 1-\varphi(0)=0, \text { and } \max _{x_{1} \neq r}\left(\left(\varphi\left(x_{1}\right)-\varphi(r)\right) /\left(x_{1}-r\right)\right) \\
& <\infty \forall r \in R \\
& A 2-(\Delta \varphi-\Delta \vartheta-\dot{r})\left(x_{1}-r\right)<0, x_{1} \neq r \\
& A 3-0<\left(\partial \vartheta\left(x_{1}\right) / \partial x_{1}\right)<k \forall x_{1} \in R \\
& A 4-(\Delta \varphi-\Delta \vartheta-\dot{r})(\dot{\varphi}(r)-\dot{\vartheta}(r))>0 \forall x_{1} \in R
\end{aligned}
$$

Here, $\Delta \varphi=\varphi\left(x_{1}\right)-\varphi(r)$ and $\Delta \vartheta=\vartheta\left(x_{1}\right)-\vartheta(r)$.
The scheme will be synthesized as follows:

$$
v=-k\left(\varphi(r)+x_{2}+\vartheta\left(x_{1}-r\right)\right),
$$

where $k \in R$ and $\vartheta: R \longrightarrow R$ makes $y=r$ a globally asymptotically stable equilibrium.

According to (30), we define an auxiliary input for system (29), which is given as follows:

$$
v=-k_{2}\left(\varphi(r)+\eta+k_{1}\left(h_{1}-r\right)\right),
$$

with $\varphi(r)=-c_{2} \sqrt{r}$ which is globally Lipchitz $(r>1)$ and $k_{2}>k_{1}>0$. In such conditions, we can suggest a structure of BC with state feedback for the state-coupled two-tank system; it is given by

$$
u=\frac{2 \sqrt{h_{2}}}{c c_{1}}\left(v+\frac{c^{2}}{2}\right) .
$$

Proof. Consider system (28) as well as the following change of variables:

$$
\left\{\begin{array}{l}
z_{1}=h_{1}-r \\
z_{2}=\eta+\varphi(r) .
\end{array}\right.
$$

To find a stabilizing control law as function of $h_{1}$ given in (28), we denote by $z_{2}$ the command variable and we define the new variable as $z_{2}^{\text {des }}=-\left[\vartheta\left(z_{1}+r\right)-\vartheta(r)\right]=-\Delta \vartheta$.

In the sequel, the variable control law with the Lyapunov control function will be defined as $W\left(z_{1}\right)=(1 / 2) z_{1}^{2}$.

The derivative of $W$ with respect to time is obtained as

$$
\begin{aligned}
\dot{W} & =z_{1} \dot{z}_{1}=z_{1}\left(\dot{h_{1}}-\dot{r}\right)=\left[\varphi\left(h_{1}\right)+\eta-\dot{r}\right] z_{1}=\left[\varphi\left(z_{1}+r\right)+z_{2}-\varphi(r)-\dot{r}\right], \\
\left.\dot{W}\right|_{z_{2}=z_{2}^{\text {des }}} & =\left[\left(\varphi\left(z_{1}+r\right)_{2}-\varphi(r)\right)-\left(\vartheta\left(z_{1}+r\right)-\vartheta(r)\right)-\dot{r}\right] z_{1}=[\Delta \varphi-\Delta \vartheta-\dot{r}] z_{1} .
\end{aligned}
$$

Then, (34) is defined negative if hypothesis A2 holds.

Now, by introducing the residue $\widetilde{z}_{2}=z_{2}-z_{2}^{\text {des }}$, system (28) can be presented as function of $z_{1}$ and $\widetilde{z}_{2}$ such as

$$
\left\{\begin{array}{l}
\dot{z}_{1}=\varphi\left(h_{1}\right)+\eta-\dot{r}=\Delta \varphi+z_{2}-\dot{r}, \\
\dot{\vec{z}}_{2}=\dot{\eta}+\dot{\varphi}(r)-\frac{\mathrm{d}\left\langle z_{2}^{\mathrm{des}}\right\rangle}{\mathrm{d} t}=v+\dot{\varphi}(r)-\frac{\mathrm{d}\left\langle z_{2}^{\mathrm{des}}\right\rangle}{d t} .
\end{array}\right.
$$

Then, we obtain

$$
\left\{\begin{array}{l}
\dot{z}_{1}=\Delta \varphi+z_{2}^{\text {des }}+\widetilde{z}_{2}-\dot{r} \\
\dot{\widetilde{z}}_{2}=v+\frac{\partial \vartheta\left(z_{1}+r\right)}{\partial z_{1}} \dot{z}_{1}+\dot{\varphi}(r)-\dot{\vartheta}(r)
\end{array}\right.
$$

From (36), we deduce

$$
\left\{\begin{array}{l}
\dot{z}_{1}=\Delta \varphi-\Delta \vartheta+\widetilde{z}_{2}-\dot{r}, \\
\dot{\tilde{z}}_{2}=v+\frac{\partial \vartheta\left(z_{1}+r\right)}{\partial z_{1}}\left(\Delta \varphi-\Delta \vartheta+\widetilde{z}_{2}-\dot{r}\right)+\dot{\varphi}(r)-\dot{\vartheta}(r) .
\end{array}\right.
$$

Now, we select the following Lyapunov control function (LCF) for system (37):

$$
V\left(z_{1}, \widetilde{z}_{2}\right)=F\left(z_{1}\right)+\frac{1}{2} \widetilde{z}_{2}^{2}
$$

where $F$ is a Lyapunov control function (CLF) of the subsystem $z_{1}$.

The derivative of the CLF given in (38) respective to the time is deduced as

$$
\dot{V}\left(z_{1}, \widetilde{z}_{2}\right)=\frac{\partial F\left(z_{1}\right)}{\partial z_{1}} \dot{z}_{1}+\widetilde{z}_{2} \dot{\bar{z}}_{2} .
$$

Therefore, we have 


$$
\begin{aligned}
\dot{V}\left(z_{1}, \widetilde{z}_{2}\right)= & \frac{\partial F\left(z_{1}\right)}{\partial z_{1}}\left(\Delta \varphi-\Delta \vartheta+\widetilde{z}_{2}-\dot{r}\right) \\
& +\widetilde{z}_{2}\left[\nu+\frac{\partial \vartheta\left(z_{1}+r\right)}{\partial z_{1}}\left(\Delta \varphi-\Delta \vartheta+\widetilde{z}_{2}-\dot{r}\right)+\dot{\varphi}(r)-\dot{\vartheta}(r)\right] .
\end{aligned}
$$

Let us consider the following function:

$$
U\left(z_{1}\right)=-\frac{\partial F\left(z_{1}\right)}{\partial z_{1}}(\Delta \varphi-\Delta \vartheta-\dot{r}) .
$$

From (40) and (41), we get

$$
\begin{aligned}
\dot{V}\left(z_{1}, \widetilde{z}_{2}\right)= & -U\left(z_{1}\right) \\
& +\widetilde{z}_{2}\left[\nu+\frac{\partial \vartheta\left(z_{1}+r\right)}{\partial z_{1}}\left(\Delta \varphi-\Delta \vartheta+\widetilde{z}_{2}-\dot{r}\right)+\frac{\partial F\left(z_{1}\right)}{\partial z_{1}}+\dot{\varphi}(r)-\dot{\vartheta}(r)\right] \frac{\Delta y}{\Delta x} .
\end{aligned}
$$

To reduce the complexity of the second term of equation (42), $F$ is chosen such that the $z_{1}$ terms inside the braces neutralize each other.

This can be done by choosing $F\left(z_{1}\right)$ such as

$$
\begin{aligned}
\frac{\partial F\left(z_{1}\right)}{\partial z_{1}} & =-\frac{\partial \vartheta\left(z_{1}+r\right)}{\partial z_{1}}\left(\Delta \varphi-\Delta \vartheta+\widetilde{z}_{2}-\dot{r}\right)-(\dot{\varphi}(r)-\dot{\vartheta}(r)), \\
F(0) & =0 .
\end{aligned}
$$

By inserting this expression in (41), we obtain

$$
\dot{V}\left(z_{1}, \widetilde{z}_{2}\right)=-U\left(z_{1}\right)+\widetilde{z}_{2}\left[v+\frac{\partial \vartheta\left(z_{1}+r\right)}{\partial z_{1}} \widetilde{z}_{2}\right] .
$$

Consider the following control law:

$$
v=-k\left(z_{2}-z_{2}^{\mathrm{des}}\right)=-k \widetilde{z}_{2} .
$$

Equation (41) becomes

$$
\dot{V}\left(z_{1}, \widetilde{z}_{2}\right)=-U\left(z_{1}\right)-\left[k-\frac{\partial \vartheta\left(z_{1}+r\right)}{\partial z_{1}} \widetilde{z}_{2}\right] \widetilde{z}_{2}^{2} .
$$

If hypothesis A3 is verified, we have $k>\left(\partial \vartheta\left(z_{1}+r\right) / \partial z_{1}\right)$, and if $\mathrm{A} 3$ and $\mathrm{A} 4$ are checked, then $U\left(z_{1}\right)>0$. definite.

Finally, it is simple to see that $\dot{V}\left(z_{1}, \widetilde{z}_{2}\right)$ is negatively

This is the end of this proof.

\section{Comparative Study of the Theoretical Analysis}

In this subsection, a comparative study for the proposed liquid-level schemes will be presented. Indeed, the comparison criteria are based on the controller design and implementation possibilities, the complexity of adjusting the controller parameters, and the stability properties of the uncertain parameters. Concerning the two schemes designs, the NTSMC expression depends on several parameters, which are very delicate to identify. It should be noted that the expression $(L+\mu) \operatorname{sign}(S)$ will be substituted by $(L+\mu)$ sat $(S)$ to minimize the chattering phenomenon with a particular choice of the parameters $L$ and $\mu$. Therefore, the parameter $\beta$ will be fixed after several simulations and practical tests. Indeed, we can deduce that this parameter has a lot of influences on the system performances and especially for its convergence speed.

The BC method is simpler than the NTSMC in terms of the computational complexity and the mathematical development. Then, the expression of the $\mathrm{BC}$ can be designed after a suitable choice of the gains $k_{1}$ and $k_{2}$.

Note that the real system parameters affect the control law performance. In fact, the values of these parameters are mainly based on the calculation of the tank's sections and the piping connection between its components. However, this calculation is generally imprecise due mainly to the measurement errors. In such conditions, the considered controllers should be robust against uncertain parameters.

\section{Results and Discussion}

6.1. Simulation Results. In this part, we present the obtained simulation results, respectively, to the NTSMC and the BC.

The numerical values of the parameters of the studied two-tank liquid-level system are given in Table 1.

Note that in the system model a bounded input voltage and bounded liquid levels have been assumed such as $0 \mathrm{~V}<u<12 \mathrm{~V}$ and $4 \mathrm{~cm}<h_{i}<30 \mathrm{~cm}$.

In what follows, two different disturbance types in tank 2 will be considered. Indeed, the disturbance scenarios are described as follows:

(i) The external disturbance is equivalent to a shock stability moment of the system; in such situation, it could be applied at each constant level: $10 \mathrm{~cm}, 16 \mathrm{~cm}$, and $8 \mathrm{~cm}$. In fact, in the simulation, this disturbance 
TABLe 1: Numerical values for physical parameters of the system.

\begin{tabular}{lcc}
\hline Physical quantity & Symbol & Numerical value \\
\hline Tank 1 diameter & $D_{1}$ & $15 \sqrt{2} \mathrm{~cm}$ \\
Tank 2 diameter & $D_{2}$ & $15 \sqrt{2} \mathrm{~cm}$ \\
Tank 1 orifice diameter & $d_{1}$ & $0.38 \mathrm{~cm}$ \\
Tank 2 orifice diameter & $d_{2}$ & $0.48 \mathrm{~cm}$ \\
Pump constant & $K_{p}$ & $7.687 \mathrm{~cm}^{3} / \mathrm{V}$ \\
Gravitational constant & $g$ & $981 \mathrm{~cm} / \mathrm{s}^{2}$ \\
\hline
\end{tabular}

is equivalent to an impulse that will be applied at the instant $t=460 \mathrm{~s}$ during $20 \mathrm{~s}$ with an amplitude equal to 1 .

(ii) The parametric disturbance has been introduced in the simulation by considering the parameter $c_{2}=0$ from $t=430 \mathrm{~s}$ to $t=515 \mathrm{~s}$.

Figures 3 and 4 show the tracking results, respectively, to the NTSMC and the BC as follows.

From Figure 3, we can see that the system response is fast (the rise time for the NTSMC is $t_{r}(\mathrm{NTSM})=50 \mathrm{~s}$ ) which causes an overshoot at each climb level of the desired trajectory $(10 \mathrm{~cm}, 16 \mathrm{~cm})$. Thus, $13 \%$ and $5.6 \%$ correspond, respectively, to the overshoot's levels $(10 \mathrm{~cm})$ and $(16 \mathrm{~cm})$. Despite the speed and the overshoot that degrade stability properties, the system maintains its stability under the two occurring disturbances.

On the other hand, from Figure 4, we can observe that the system response via the $\mathrm{BC}$ is slow (the rise time for the $\mathrm{BC}$ is $\left.t_{r}(\mathrm{BC})=100 \mathrm{~s}\right)$ which leads to static errors at each change of the desired liquid level. Therefore, 27\%, 20\%, and $45 \%$ are the errors in percent relative, respectively, to the desired liquid level $(10 \mathrm{~cm}, 16 \mathrm{~cm}$, and $8 \mathrm{~cm})$.

Finally, we can conclude that the tracking results from the different schemes are suitable and satisfactory. However, the NTSMC allows obtaining more performant results compared to the BC.

Figure 5 represents the NTSMC and BC schemes signals in presence of external disturbances.

Figure 5 shows that the obtained signals are regulated and their amplitude remains within a permissible and bounded limit. In addition, we can see that the BC is more economical than the NTSMC since the maximum and average values of the $\mathrm{BC}$ signal are smaller than those of the NTSMC.

According to the results presented in Figure 5, the motopump voltage takes its maximum value $(12 \mathrm{~V})$ to reach the liquid levels $(10 \mathrm{~cm}$ and $16 \mathrm{~cm})$. However, to go from $16 \mathrm{~cm}$ to $8 \mathrm{~cm}$, the moto-pump stops or operates in the dead zone leaving tank 2 free to empty. These results are similar for both controls. In addition, the voltage values remain equal to $(7.1 \mathrm{~V}, 9 \mathrm{~V}$, and $6.4 \mathrm{~V})$ for the NTSMC and $(6.7 \mathrm{~V}, 8.4 \mathrm{~V}$, and $6 \mathrm{~V})$ for the $\mathrm{BC}$, respectively, to the regarding bearings. Thus, we can conclude that the $\mathrm{BC}$ is less aggressive than the NTSMC. Although all schemes have a positive reaction for disturbances rejection, the NTSMC makes 65 sto reject the external disturbances and the $\mathrm{BC}$ makes56 for the same task with a small difference of $9 \mathrm{~s}$.

\subsection{Experimental Results}

6.2.1. Test Bench Description. The practical two-tank liquidlevel system is presented in Figure 6. This system exists in the Laboratory Study of Industrial Systems and Renewable Energies "LAS2E" at the National Engineers School of Monastir, Tunisia. In fact, the electromechanical part of the system is made up of the tanks, the power card motor pumps, and conditioning cards for pressure sensors. The synthesized schemes will be implemented by using blocks in MATLAB/Simulink environment combined with the interface real time associated with the data acquisition board which is based on the Arduino Mega microcontroller.

Note that the schemes are made in the same environment and the same conditions. In addition, the initial liquid levels $h_{1}$ and $h_{2}$ are always equal to $4 \mathrm{~cm}$ and the conversion voltage/level of the two voltages measured from the two pressure sensors with their instrumentation amplifiers is made through multiplication by the coefficients $a_{1}=9.3458 \mathrm{~cm} \cdot \mathrm{V}^{-1}$ and $a_{2}=8.5106 \mathrm{~cm} \cdot \mathrm{V}^{-1}$. Finally, the numerical parameters values are $k_{1}=0.032$ and $k_{2}=0.11$ for the BC and $L=1.1, \mu=1.3, \beta=17, p=5$, and $q=3$ for the NTSMC.

Remark 2. The simulation tests allow obtaining approximate parameters for all schemes. However, the best values will be deduced from several experimental testings.

We suppose that the system meets two types of disturbances. Indeed, the disturbance scenarios are explained as follows:

The external disturbance has been generated by suddenly adding at the instants $150 \mathrm{~s}, 500 \mathrm{~s}$, and $800 \mathrm{~s}$ a water quantity in the tank 2.

The parametric disturbance will be affected by varying the value of $c_{2}$. In such situation, we close the orifice related to $c_{2}$ for a few seconds at the same instants that have been chosen for the external disturbances.

6.2.2. Comparison of the Measurement Results. The experimental results are illustrated in Figures $7-10$ as follows which represent, respectively, the NTSMC tracking and its impact of the external disturbance, the NTSMC tracking and its impact on the parameter variation, the BC tracking and its impact on the external disturbance, and the $\mathrm{BC}$ tracking and its impact on the parameter variation. 


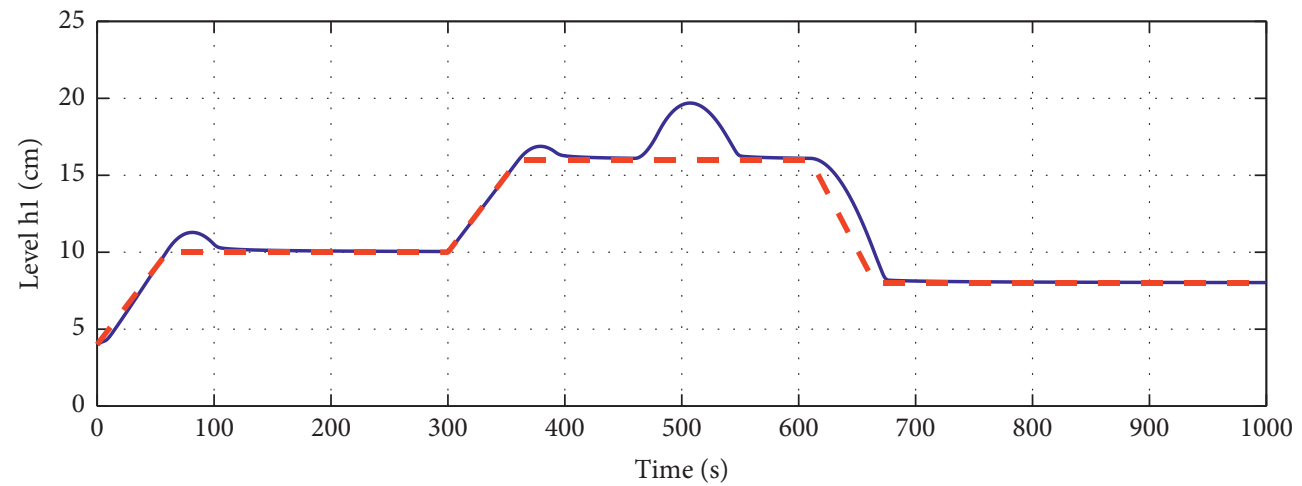

(a)

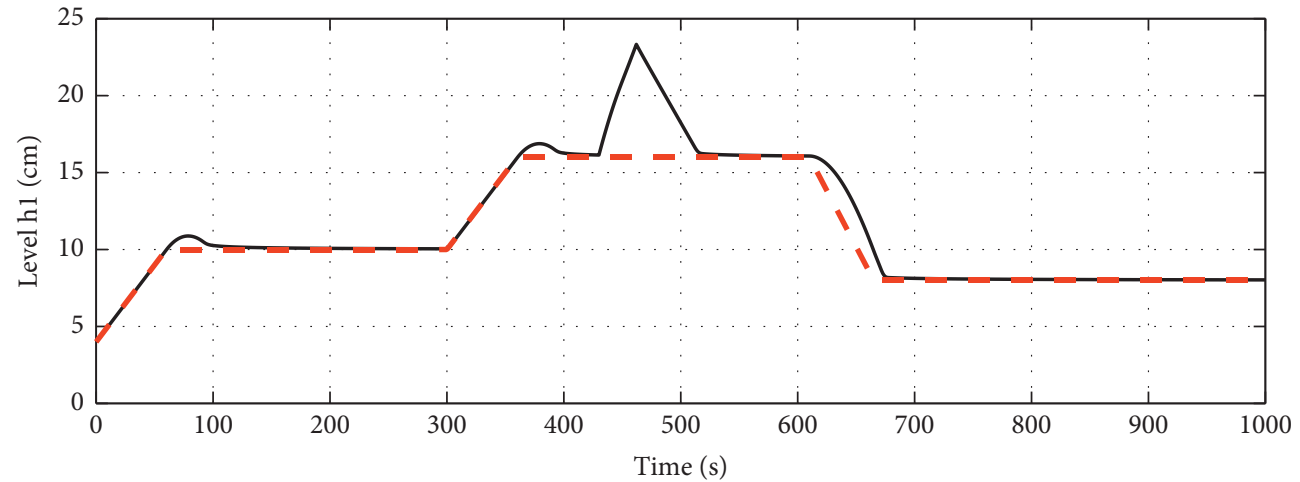

(b)

FIGURE 3: NTSMC tracking result and its impact on disturbances. (a) With impact of the external disturbance; (b) with impact of the parameter variation.

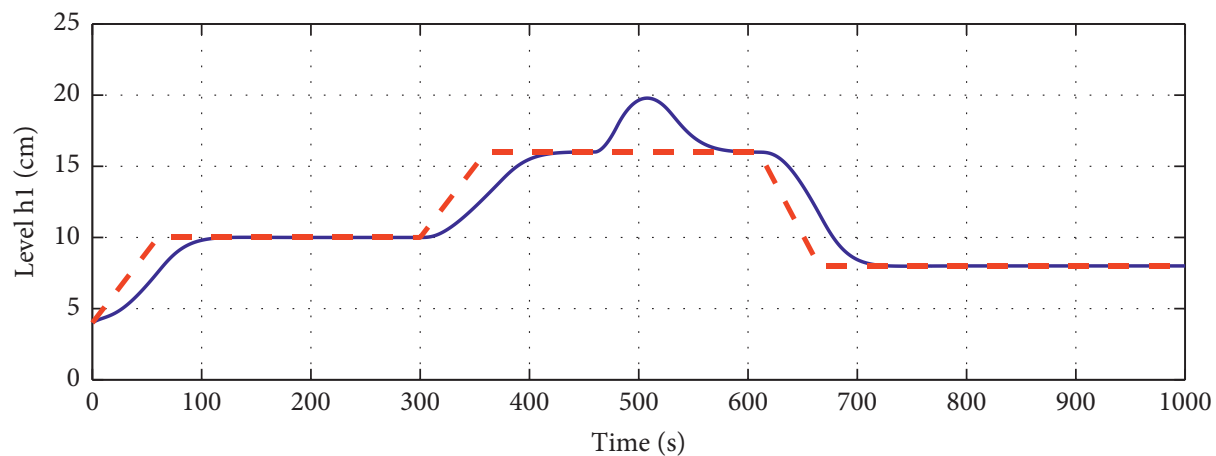

(a)

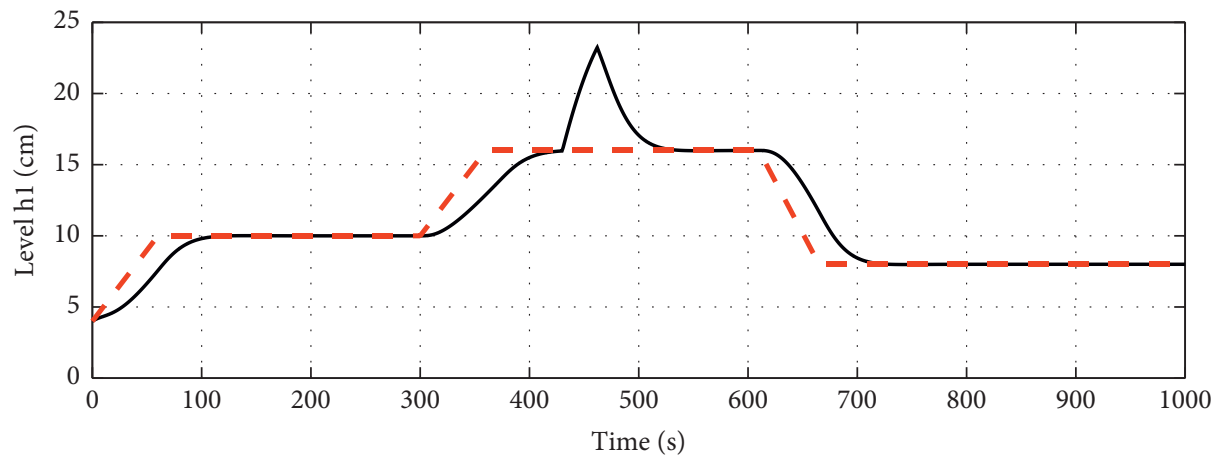

(b)

FIGURE 4: BC tracking result and its impact on disturbances. (a) With impact of the external disturbance; (b) with impact of the parameter variation. 


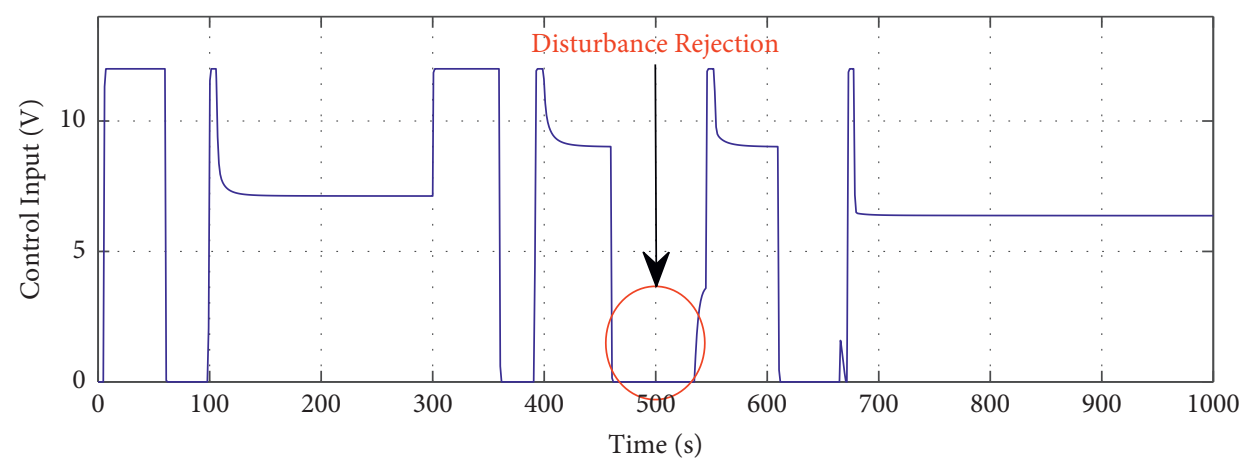

(a)

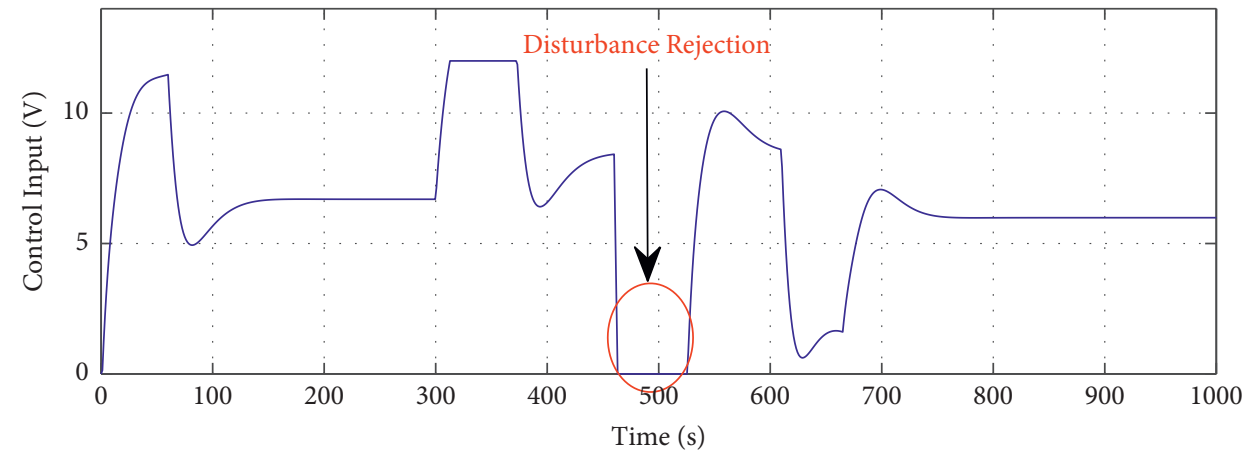

(b)

FIGURE 5: NTSMC and BC signals with external disturbance rejection. (a) NTSM voltage; (b) backstepping voltage.

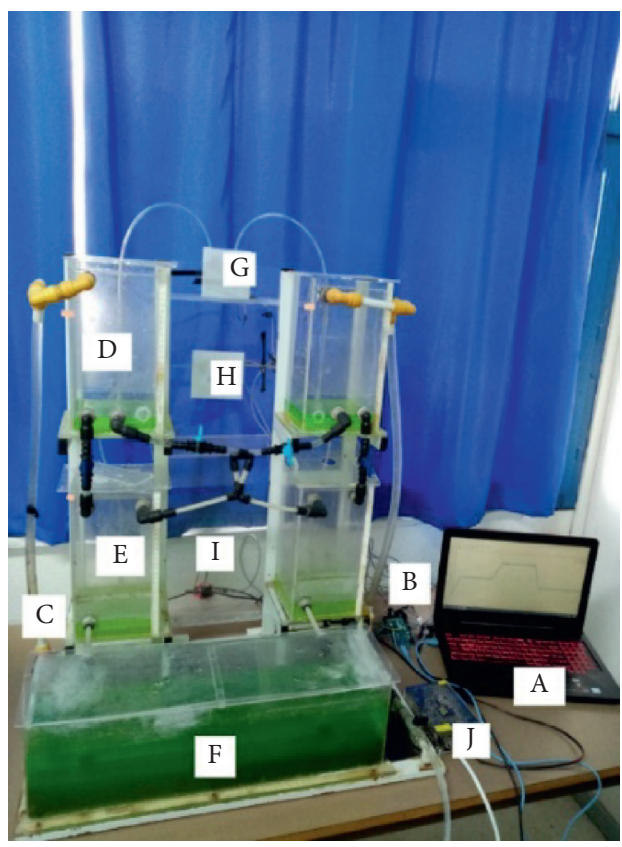

Figure 6: Experimental setup. A: control desk; B: Arduino; C: pump with maximum voltage rating 12 V; D: tank 2; E: tank 1; F: basin; G: liquid-level sensor for tank 2; H: liquid-level sensor for tank 1; I: motor driver board; J: $\pm 12 \mathrm{~V}$ supply voltage.

From Figures 7 and 8, the following overshoot values 4\%, $5 \%$, and $10 \%$ are, respectively, obtained at levels $10 \mathrm{~cm}$, $16 \mathrm{~cm}$, and $8 \mathrm{~cm}$. This result allows us to deduce that the corresponding system has satisfactory performance in terms of tracking, stability, fastness, and robustness when the
NTSMC has been applied. Therefore, the maximum overshoot percentage of NTSMC is equal to $\mathrm{PO}_{\max }(\mathrm{NTSMC})=$ $10 \%$ and the rise time for NTSMC is $t_{r}($ NTSMC) $=30 \mathrm{~s}$.

It can be shown from Figures 9 and 10 that there is a notable static error. The following overshoot values $10 \%$, 


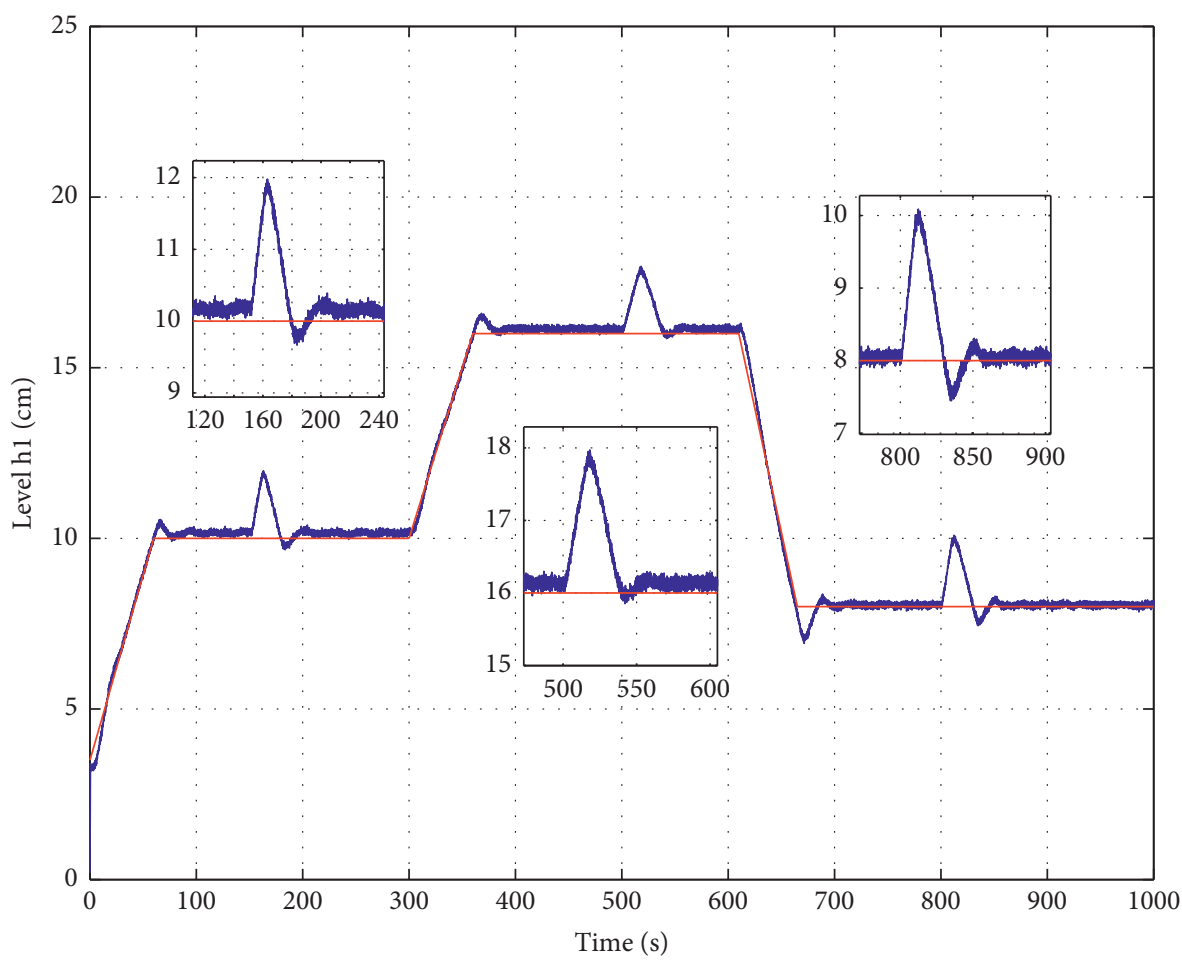

FIGURE 7: Experiment result for NTSMC tracking and its impact on external disturbance.

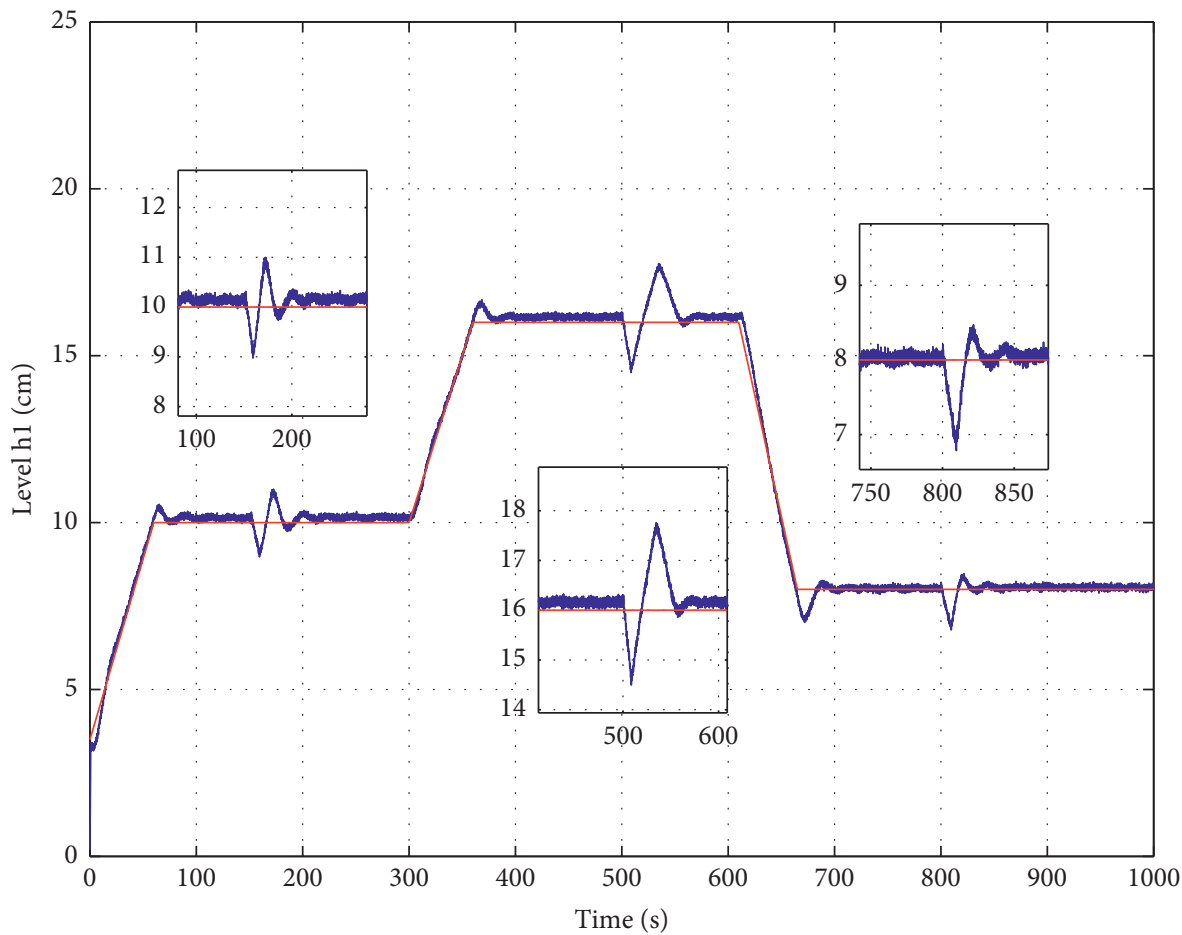

Figure 8: Experimental result for NTSMC tracking and its impact on parameter variation. 


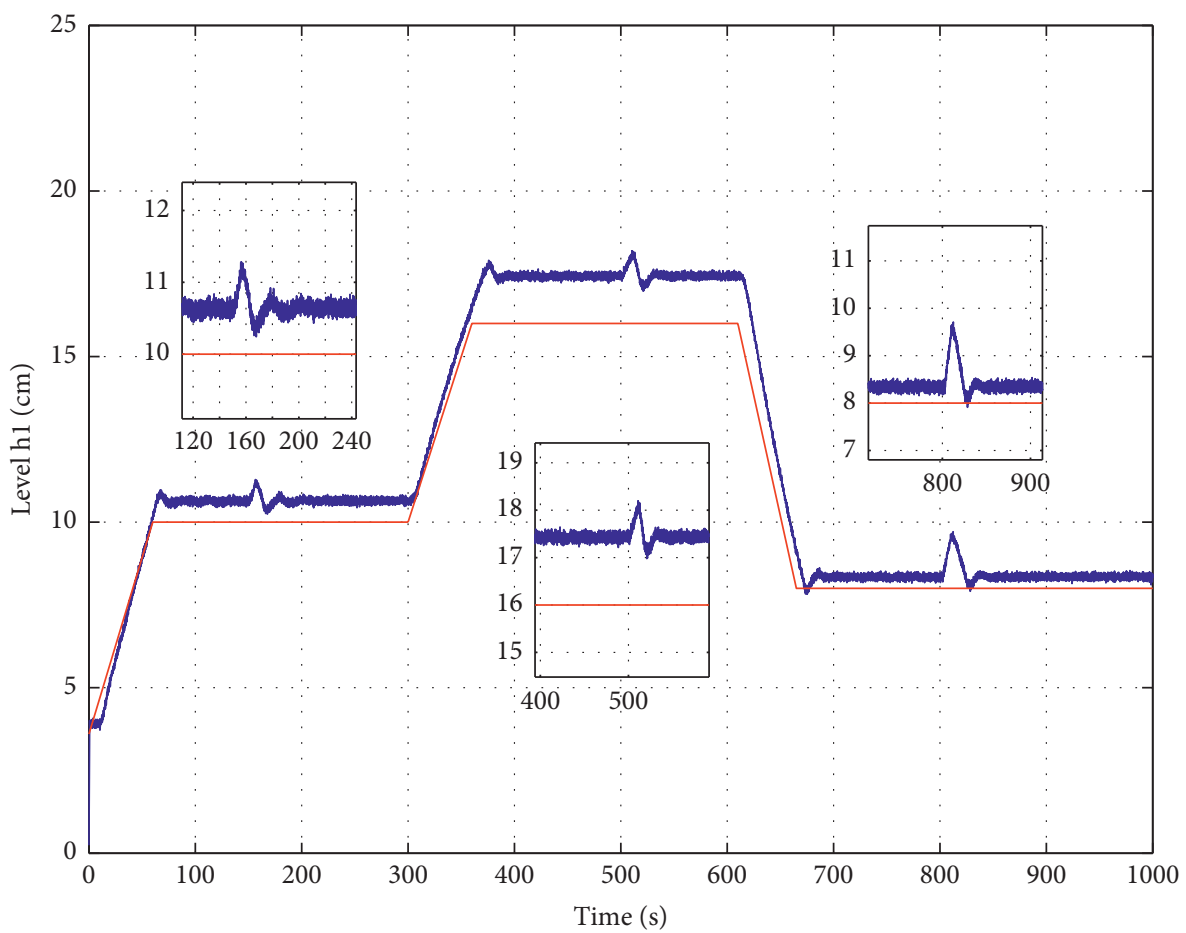

FIgURE 9: Experimental result for BC tracking and its impact on external disturbance.

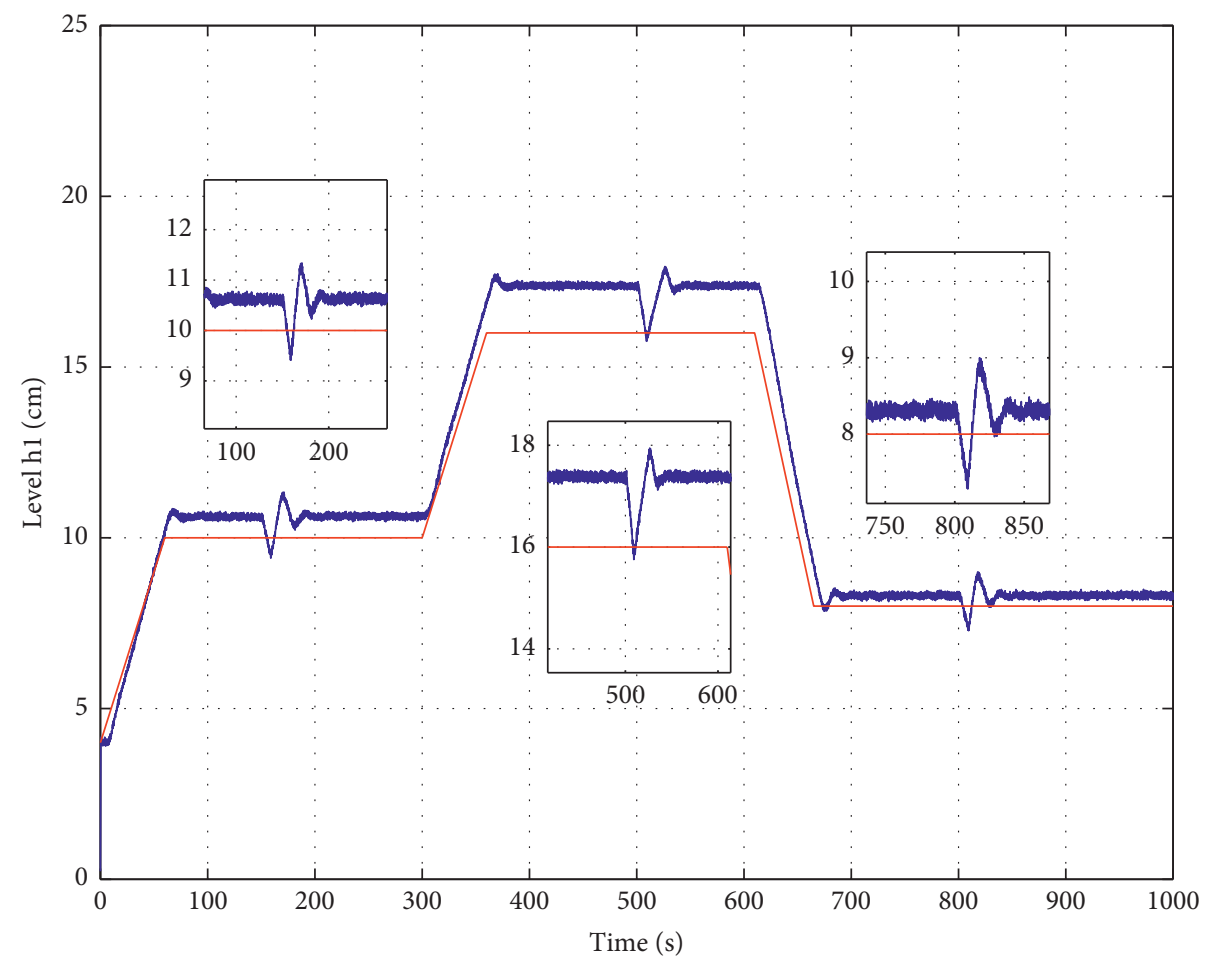

FIGURE 10: Experimental result of tracking by BC and impact on parameter variation. 
$12.5 \%$, and $0.6 \%$ are, respectively, obtained at levels $10 \mathrm{~cm}$, $16 \mathrm{~cm}$, and $8 \mathrm{~cm}$. Then, the maximum overshoot percentage via $\mathrm{BC}$ equals $\mathrm{PO}_{\max }(\mathrm{BC})=12.5 \%$ and the rise time for $\mathrm{BC}$ is $t_{r}(\mathrm{BC})=45 \mathrm{~s}$.

In what follows, an integrator action will be added to the $\mathrm{BC}$ to eliminate the static error. In fact, in the recent years, the integrator backstepping control (IBC) has gained much focus since it provides a framework for attacking many electromechanical control troubles like the state-coupled two-tank system.

For systematic condition, requested control structure adjustments such as compensation for parametric uncertainty or eliminating state measurements can be one of the major benefits of the IBC family of control design tools. This would be confirmed by the results given in Figures 11 and 12.

From Figures 11 and 12, the integral action avoids the static error. Despite the fact that the presence of this action degrades the stability performance, the system has a good tracking and a performed robustness in the presence of an external disturbance and parameters variation.

Accordingly, the system response becomes rapid which leads to overshoots at each level change. The following overshoot values $8.5 \%, 5 \%$, and $23 \%$ correspond, respectively, to the levels $10 \mathrm{~cm}, 16 \mathrm{~cm}$, and $8 \mathrm{~cm}$.

Thus, the IBC maximum overshoot percentage equals $\mathrm{PO}_{\max }(\mathrm{IBC})=23 \%$ and the rise time of the IBC is $t_{r}(\mathrm{IBC})=25 \mathrm{~s}$.

The control voltages in the presence of external disturbance and parameter variation for the NTSM, B, and IB controllers are given, respectively, in Figures 13-15.

The voltage provided by the NTSMC scheme is shown in Figure 13. Indeed, the voltage is equal to $10 \mathrm{~V}$ at the first desired slope. Once the level reaches $10 \mathrm{~cm}$, this voltage is kept at constant value which equals $5.5 \mathrm{~V}$. At the second rise, the voltage is equal to $10.8 \mathrm{~V}$. When the level reaches $16 \mathrm{~cm}$, the voltage settles approximately at $7.5 \mathrm{~V}$.

By some comparisons in terms of the energy consumption for three applied schemes, we defined the maximum and the minimum voltage values $U_{\max }$ and $U_{\min }$ for each controller such as $U_{\max }($ NTSMC $)=10.8 \mathrm{~V}$ and $U_{\text {min }}(\mathrm{NTSMC})=0 \mathrm{~V}$.

Figures 14 and 15 present, receptively, the $\mathrm{BC}$ voltage with disturbances rejection and the IB voltage with disturbances rejection as follows.

It is seen from Figure 15 that the integral action slightly increases the moto-pump voltage so that $h_{1}$ liquid level reaches 10 and $16 \mathrm{~cm}$ desired values. For the change of level from 16 to $8 \mathrm{~cm}$, the moto-pump ceased. This can be proved by the following values:

For the IBC, the voltage is $10.3 \mathrm{~V}$ at the first desired slope. Once the level reaches $10 \mathrm{~cm}$, this voltage is kept at a constant value equal to $5.8 \mathrm{~V}$. At the second rise, the voltage is $11.3 \mathrm{~V}$. When the level reaches $16 \mathrm{~cm}$, the voltage settles approximately at $7.4 \mathrm{~V}$.

At the third negative slope, the voltage is equal to $0 \mathrm{~V}$ which is explained by the free emptying of $h_{2}$ tank. Then, the voltage rises to $11.6 \mathrm{~V}$ and it settles at $5.4 \mathrm{~V}$ when the level reaches $8 \mathrm{~cm}$.
In such situation, the maximum and the minimum voltage values are $U_{\max }(\mathrm{IBC})=11.6 \mathrm{~V}$ and $U_{\min }(\mathrm{IBC})$ $=0 \mathrm{~V}$. As it is shown in Figure 14, the maximum and the minimum voltage values are $U_{\max }(\mathrm{BC})=11 \mathrm{~V}$ and $U_{\min }(\mathrm{BC})=0 \mathrm{~V}$.

Concerning the external disturbance, we notice that the three schemes react to preserve the system stability and reject any negative interference, which proves their robustness. These reactions are indicated in Figures 13-15.

The following values $35 \mathrm{~s}, 70 \mathrm{~s}$ and $50 \mathrm{~s}$ represent, respectively, the external disturbance rejection durations for the BC, IBC, and NTSMC.

In presence of the external disturbance, the pump interrupts instantly, which is explained by the increase of $h_{2}$ level due to the external liquid addition. Then, after the disturbance fading, the pump signal resumes its previous voltage. By closing the orifice output in tank 2 for a few seconds, a variation of $c_{2}$ parameter occurs.

As a control response towards the parameter variation, the pump voltage increases which is explained by $h_{1}$ tank demand for liquid from $h_{2}$ tank. By cancelling this variation, the pump voltage decreases since $h_{2}$ level has increased. Then, the pump signal resumes its previous voltage.

By some conclusions, the NTSMC signal is less aggressive than those of BC and IBC ones. This means that NTSMC is more economical in terms of energy consumption.

On the other hand, the tracking error as a percent will be represented to master the tracking quality. Figure 16 shows the respective tracking errors of the NTSMC, BC, and IBC strategies. The tracking error is calculated according to (47).

To confirm the best tracking results extracted by the three schemes, we will compare the different tracking errors such as $E(\mathrm{NTSM})<E(\mathrm{IBC})<E(\mathrm{BC})$.

We notice that the error related to NTSMC and IBC is less than that of BC. During disturbances, the tracking error increases to what is expected.

The error and the overshoot expressions will be given, respectively, in the following equations:

$$
\begin{aligned}
\text { error } \% & =\frac{\left|h_{1}-r\right|}{r} \times 100, \\
D \% & =\frac{y_{\max }-y_{\infty}}{y_{\infty}} \times 100 .
\end{aligned}
$$

Figure 16 shows the NTSMC, BC, and IBC liquid-level tracking errors in the presence of the external disturbance.

The theoretical and practical obtained results are summarized in Table 2.

Remark 3. The obtained results will be compared to those illustrated in [3]. In fact, in [3] the authors present some performance criteria for the backstepping scheme applied to the two-tank system for the tracking problem to the same desired trajectory as in the considered case.

Remark 4. Note that the SMC and BC have been combined to build a more efficient control named backstepping sliding mode control (BSMC) [42] that can be suggested to be applied later. 


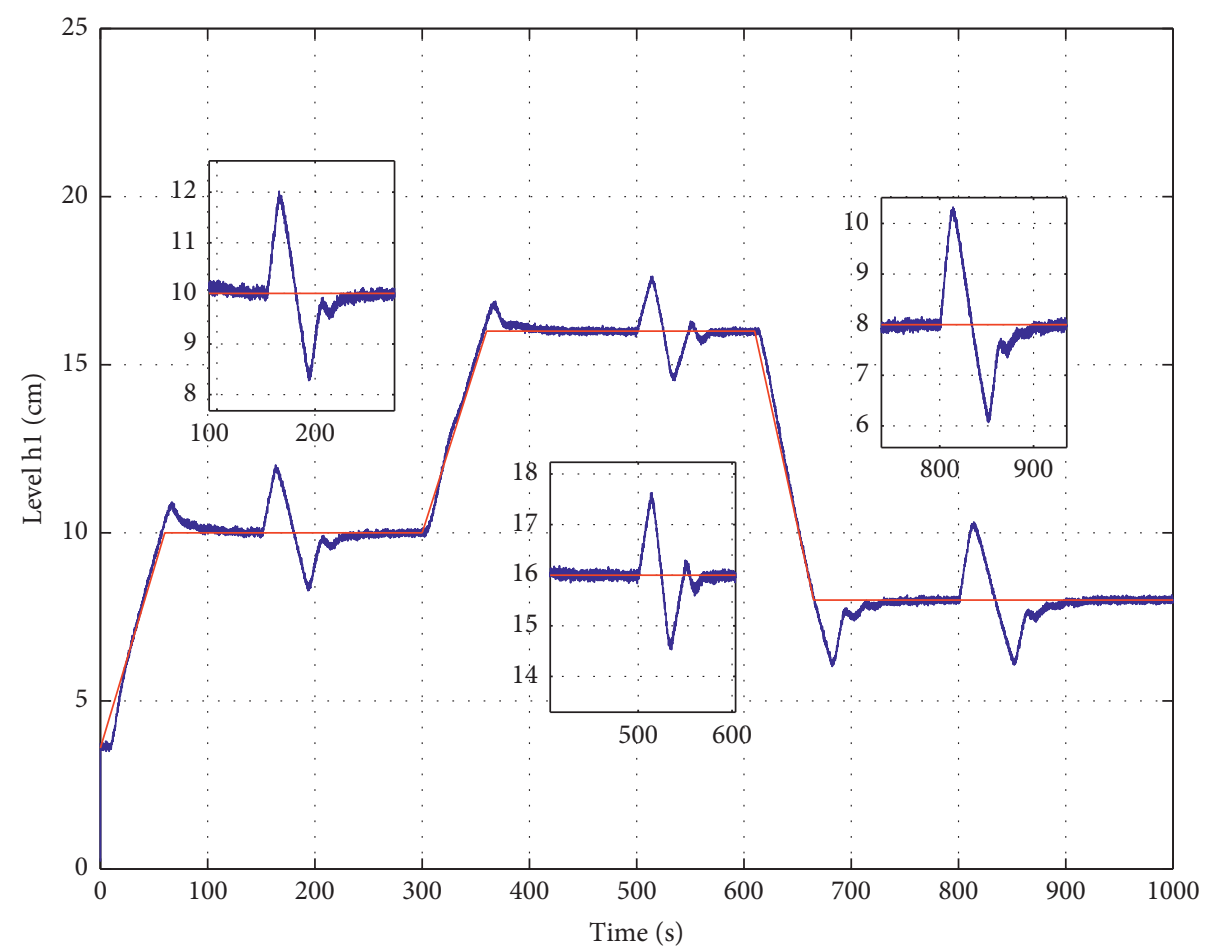

FIGURE 11: Experimental result for IBC tracking and its impact on external disturbance.

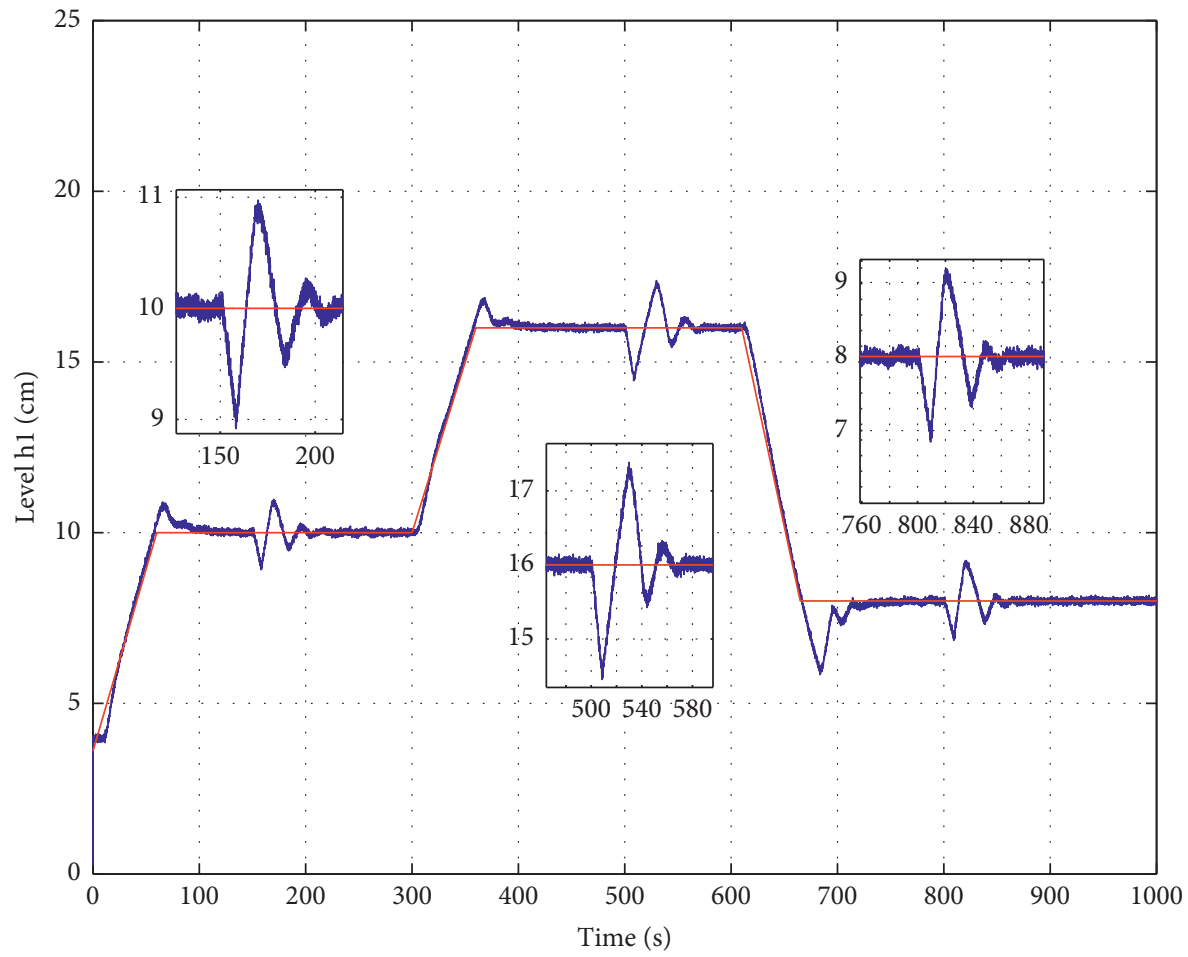

FIGURE 12: Experimental result for IBC tracking and its impact on parameter variation. 


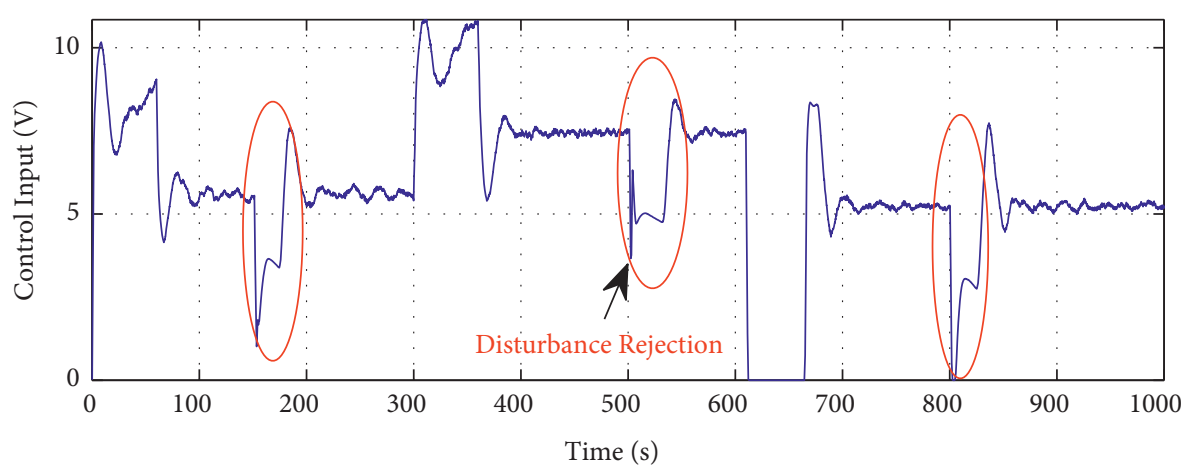

(a)

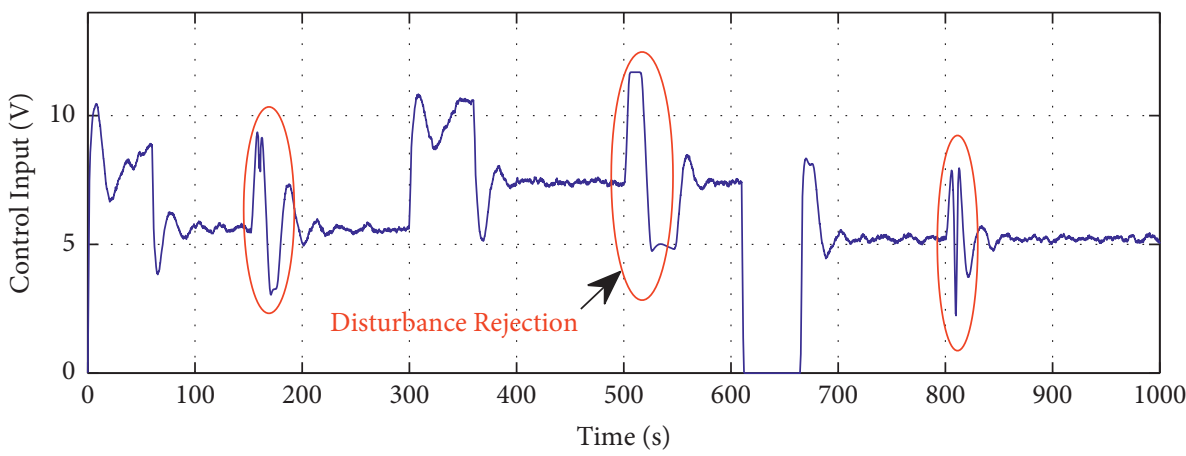

(b)

FIGURE 13: NTSM voltage with disturbances rejection. (a) With the reaction of rejecting the external disturbance; (b) with the reaction of rejecting the parameter variation.

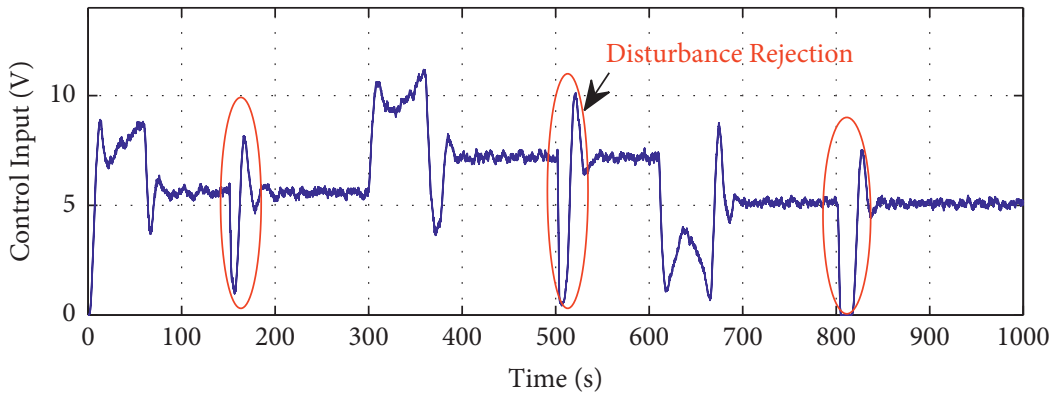

(a)

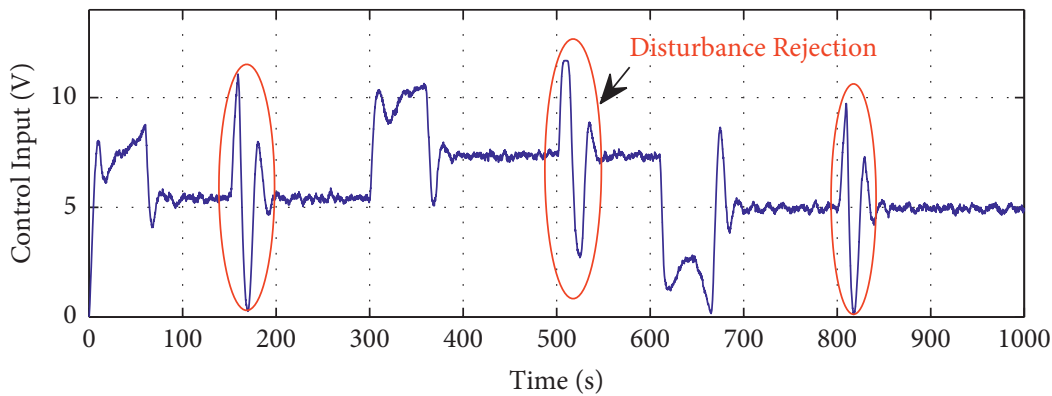

(b)

FIGURE 14: BC voltage with disturbances rejection. (a) With the reaction of reject the external disturbance; (b) with the reaction of rejecting the parameter variation. 


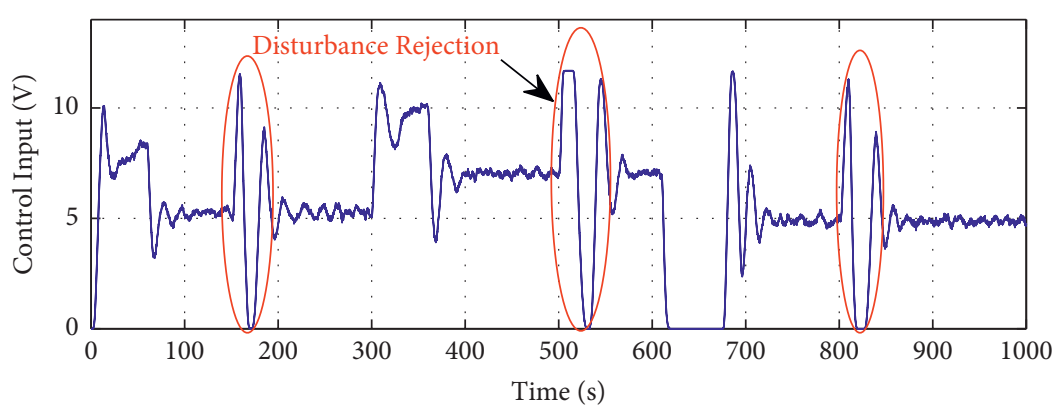

(a)

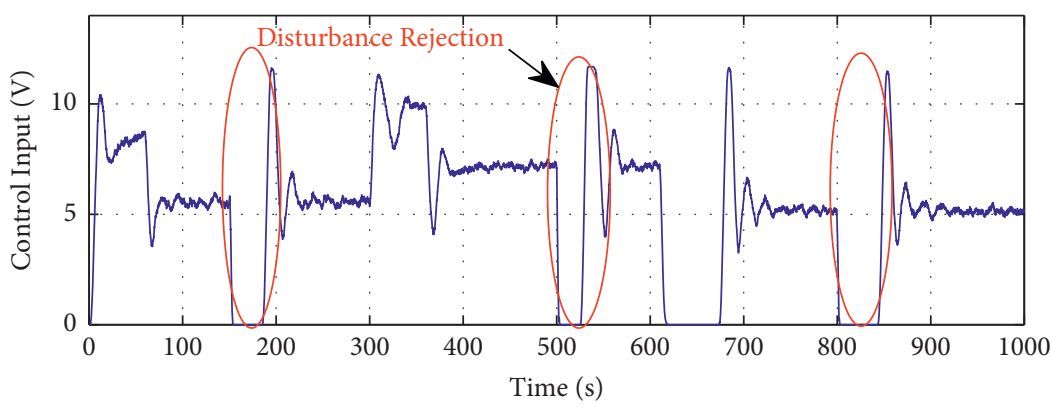

(b)

FIGURE 15: IB voltage with disturbances rejection. (a) With the reaction of rejecting the external disturbance; (b) with the reaction of rejecting the parameter variation.

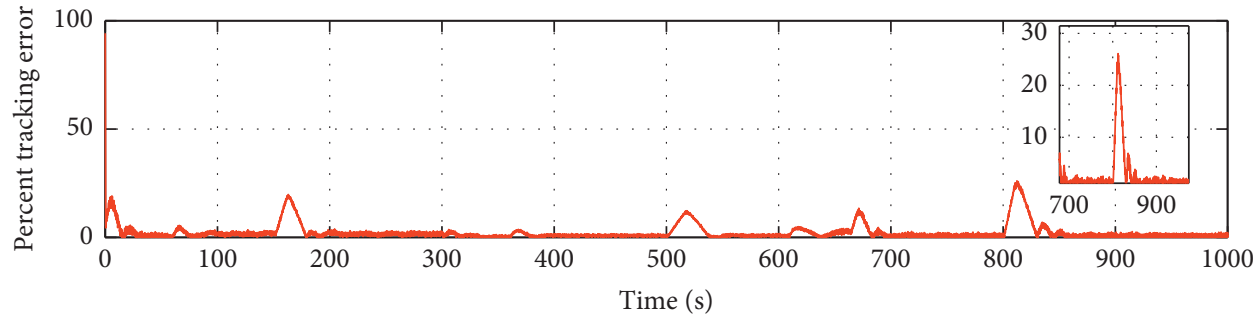

(a)

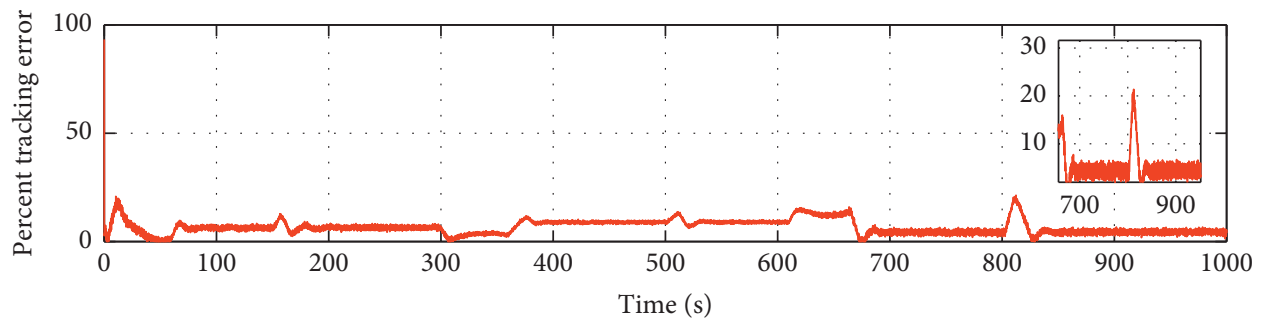

(b)

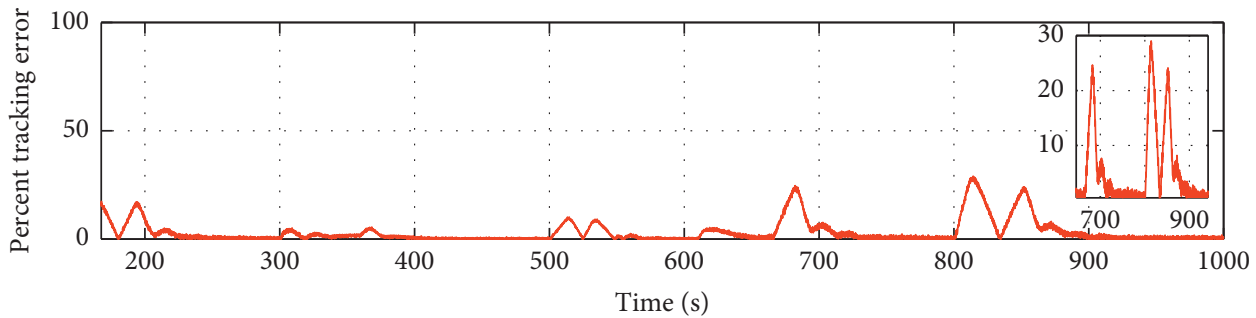

(c)

FIGURE 16: NTSMC, BC, and IBC liquid-level tracking errors in the presence of the external disturbance. (a) Tracking error related to NTSMC, (b) tracking error related to BC, and (c) tracking error related to IBC. 
TABLE 2: Comparison summary.

\begin{tabular}{|c|c|c|c|c|}
\hline Comparison criteria & $\mathrm{BC}$ & IBC & NTSMC & $\mathrm{BC}[3]$ \\
\hline Relative stability & Good & Medium & Excellent & Good \\
\hline Robustness concerning uncertain parameters & Medium & Medium & Good & Excellent \\
\hline Precision performance & Medium & Good & Excellent & Good \\
\hline Possibilities of controller design & Low & Hard & Hard & Low \\
\hline Complexity of parameters tuning & Medium & Medium & Hard & Medium \\
\hline Complexity of implementation & Low & Low & Hard & Low \\
\hline Disturbance rejection & Excellent & Good & Excellent & - \\
\hline Overshoot $(\%)$ & $\leq 2$ & 23 & 10 & - \\
\hline Tracking error (\%) & 5 & $\leq 1$ & $\leq 2$ & - \\
\hline
\end{tabular}

\section{Conclusion and Future Works}

In this paper, nonlinear controllers have been designed for the purpose of a precise liquid-level tracking in a statecoupled two-tank system by using the $\mathrm{BC}$, the IBC, and the NTSMC techniques. Indeed, it has been proved that the corresponding closed loop process for all the considered schemes is stable.

To illustrate the enhanced performance of the proposed nonlinear controllers, we started with introducing various simulations and experimental results; then we proceeded with a detailed comparison of three different schememethods. These schemes are established based on their dynamic behavior, their stability, and their robustness properties. Particularly, this study has been compared to other related works presenting the practical results for the $\mathrm{BC}$. The simulation results satisfy the performance and is proved by practice, which values the results given in this work.

Future research will extend the results of this work for MIMO systems. Future research will extend the results of this work for MIMO systems and implement an observer in cases when the state variables system is not all available. In addition, the estimation or the modeling of a physical model can be approximate by using type-2 fuzzy logic $[43,44]$.

\section{Data Availability}

No data were used to support this study.

\section{Conflicts of Interest}

The authors declare no conflicts of interest.

\section{References}

[1] R. N. Bateson, Introduction to Control System Technology, Prentice-Hall, Upper Saddle River, NJ, USA, 1999.

[2] I. S. Isa, B. C. C. Meng, Z. Saad, and N. A. Fauzi, "Comparative study of PID controlled modes on automatic water level measurement system," in Proceedings of the 7th International Colloquium on Signal Processing and its Applications, pp. 237-242, Penang, Malaysia, March 2011.

[3] H. Gouta, S. H. Said, and F. M'Sahli, "Predictive and backstepping control of double tank process: a comparative study," IETE Technical Review, vol. 33, no. 2, pp. 137-147, 2016.

[4] H. Gouta, S. H. Said, N. Barhoumi, and F. M'Sahli, "Observerbased backstepping controller for a state-coupled two-tank system," IETE Journal of Research, vol. 61, no. 3, pp. 259-268, 2015.

[5] X. Lin, K. Liang, H. Li, Y. Jiao, and J. Nie, "Robust finite-time $\mathrm{H}$-infinity control with transients for dynamic positioning ship subject to input delay," Mathematical Problems in Engineering, vol. 2018, Article ID 2838749, 17 pages, 2018.

[6] A. Gaaloul and F. M'Sahli, "Output feedback higher order sliding mode controller," International Review of Automatic Control, vol. 4, no. 1, pp. 32-39, 2011.

[7] M. Kermani and A. Sakly, "M-matrix-based robust stability and stabilization criteria for uncertain switched nonlinear systems with multiple time-varying delays," Mathematical Problems in Engineering, vol. 2021, Article ID 8871603, 17 pages, 2021.

[8] M. Kermani and A. Sakly, "Pole assignment stabilization for a class of switched nonlinear time-varying delay systems," ISA Transactions, vol. 106, pp. 138-151, 2020.

[9] Q. H. Do and J. F. Chen, "A neuro-fuzzy approach in the classification of students' academic performance," Сomputational Intelligence and Neuroscience, vol. 2013, Article ID 179097, 7 pages, 2013.

[10] M. T. Alam, Z. H. Khan, P. Charan, and M. A. Ansari, "Level control of coupled tanks system using feedback linearization control theory," International Journal for Scientific Research and Development, vol. 3, pp. 60-63, 2015.

[11] W. Zhou, K. Yin, R. Wang, and Y. E. Wang, "Design of attitude control system for UAV based on feedback linearization and adaptive control," Mathematical Problems in Engineering, vol. 2014, Article ID 492680, 8 pages, 2014.

[12] C. Huang, E. Canuto, and C. Novara, "The four-tank control problem: comparison of two disturbance rejection control solutions," ISA Transactions, vol. 71, pp. 252-271, 2017.

[13] I. Garrido, A. J. Garrido, J. A. Romero, E. Carrascal, G. Sevillano-Berasategui, and O. Barambones, "Low effort nuclear fusion plasma control using model predictive control laws," Mathematical Problems in Engineering, vol. 2015, Article ID 527420, 8 pages, 2015.

[14] D. Q. Mayne, J. B. Rawlings, C. V. Rao, and P. O. M. Scokaert, "Constrained model predictive control: stability and optimality," Automatica, vol. 36, no. 6, pp. 789-814, 2000.

[15] H. Pan, H. Wong, V. Kapila, and M. S. De Queiroz, "Experimental validation of a nonlinear backstepping liquid level controller for a state coupled two tank system," Control Engineering Practice, vol. 13, no. 1, pp. 27-40, 2005.

[16] Y. Wei, Y. Wang, C. K. Ahn, and D. Duan, "IBLF-based finitetime adaptive fuzzy output-feedback control for uncertain mimo nonlinear state-constrained systems," IEEE Transactions on Fuzzy Systems, vol. 2020, Article ID 3021733, 13 pages, 2020. 
[17] V. I. Utkin and H. C. Chang, "Sliding mode control on electro-mechanical systems," Mathematical Problems in Engineering, vol. 2002, Article ID 635132, 23 pages, 2002.

[18] X. Cao, P. Shi, Z. Li, and M. Liu, "Neural-network-based adaptive backstepping control with application to spacecraft attitude regulation," IEEE transactions on neural networks and learning systems, vol. 29, no. 9, pp. 4303-4313, 2017.

[19] X. Huo, M. Huo, and H. R. Karimi, "Attitude stabilization control of a quadrotor UAV by using backstepping approach," Mathematical Problems in Engineering, vol. 2014, Article ID 749803, 9 pages, 2014.

[20] R. Li, T. Li, R. Bu, Q. Zheng, and C. L. Chen, "Active disturbance rejection with sliding mode control-based course and path following for underactuated ships," Mathematical Problems in Engineering, vol. 2013, Article ID 743716, 9 pages, 2013.

[21] B. Xu and P. Zhang, "Composite learning sliding mode control of flexible-link manipulator," Complexity, vol. 2017, Article ID 9430259, 9 pages, 2017.

[22] M. Nasiri, S. Mobayen, and Q. M. Zhu, "Super-twisting sliding mode control for gearless PMSG-based wind turbine," Complexity, vol. 2019, Article ID 6141607, 15 pages, 2019.

[23] N. F. Al-Muthairi and M. Zribi, "Sliding mode control of a magnetic levitation system," Mathematical Problems in Engineering, vol. 2014, Article ID 657503, 15 pages, 2014.

[24] J. Xu, M. Wang, and L. Qiao, "Dynamical sliding mode control for the trajectory tracking of underactuated unmanned underwater vehicles," Ocean Engineering, vol. 105, pp. 54-63, 2015.

[25] Y. Wang, X. Xie, M. Chadli, S. Xie, and Y. Peng, "Sliding mode control of fuzzy singularly perturbed descriptor systems," IEEE Transactions on Fuzzy Systems, vol. 2020, Article ID 2998519, 12 pages, 2020.

[26] Y. Wang, Y. Xia, H. Li, and P. Zhou, "A new integral sliding mode design method for nonlinear stochastic systems," Automatica, vol. 90, pp. 304-309, 2018.

[27] F. Abu Khadra and J. Abu Qudeiri, "Second order sliding mode control of the coupled tanks system," Mathematical Problems in Engineering, vol. 2015, Article ID 167852, 9 pages, 2015.

[28] M. Chen, Q.-X. Wu, and R.-X. Cui, "Terminal sliding mode tracking control for a class of SISO uncertain nonlinear systems," ISA Transactions, vol. 52, no. 2, pp. 198-206, 2013.

[29] J. Liu and X. Wang, "Terminal sliding mode control, advanced sliding mode control for mechanical systems," in Advanced Sliding Mode Control for Mechanical SystemsSpringer, Berlin, Heidelberg, 2011.

[30] X. Wang, J. Liu, and K.-Y. Cai, "Tracking control for VTOL aircraft with disabled IMUs," International Journal of Systems Science, vol. 41, no. 10, pp. 1231-1239, 2010.

[31] X. Xinghuo Yu and M. Man Zhihong, "Fast terminal slidingmode control design for nonlinear dynamical systems," IEEE Transactions on Circuits and Systems I: Fundamental Theory and Applications, vol. 49, no. 2, pp. 261-264, 2002.

[32] M. Labbadi and M. Cherkaoui, "Robust integral terminal sliding mode control for quadrotor UAV with external disturbances," International Journal of Aerospace Engineering, vol. 2019, Article ID 2016416, 10 pages, 2019.

[33] S. Peng and W. Shi, "Adaptive fuzzy integral terminal sliding mode control of a nonholonomic wheeled mobile robot," Mathematical Problems in Engineering, vol. 2017, Article ID 3671846, 12 pages, 2017.

[34] M. D. Tran and H. J. Kang, "Nonsingular terminal sliding mode control of uncertain second-order nonlinear systems,"
Mathematical Problems in Engineering, vol. 2017, Article ID 181737, 12 pages, 2017.

[35] S. Y. Chen and F. J. Lin, "Robust nonsingular terminal slidingmode control for nonlinear magnetic bearing system," IEEE Transactions on Control Systems Technology, vol. 19, no. 3, pp. 636-643, 2010.

[36] L. Qiao and W. Zhang, "Trajectory tracking control of AUVs via adaptive fast nonsingular integral terminal sliding mode control," IEEE Transactions on Industrial Informatics, vol. 16, no. 2, pp. 1248-1258, 2019.

[37] M. B. R. Neila and D. Tarak, "Adaptive terminal sliding mode control for rigid robotic manipulators," International Journal of Automation and Computing, vol. 8, no. 2, pp. 215-220, 2011.

[38] P. Wang, D. Zhang, and B. Lu, "Trajectory tracking control for chain-series robot manipulator: robust adaptive fuzzy terminal sliding mode control with low-pass filter," International Journal of Advanced Robotic Systems, vol. 17, no. 3, pp. 1-17, 2020.

[39] H. Komurcugil, "Adaptive terminal sliding-mode control strategy for DC-DC buck converters," ISA Transactions, vol. 51, no. 6, pp. 673-681, 2012.

[40] L. Qiao and W. Zhang, "Adaptive non-singular integral terminal sliding mode tracking control for autonomous underwater vehicles," IET Control Theory \& Applications, vol. 11, no. 8, pp. 1293-1306, 2017.

[41] M. Krstic, D. Fontaine, P. V. Kokotovic, and J. D. Paduano, "Useful nonlinearities and global stabilization of bifurcations in a model of jet engine surge and stall," IEEE Transactions on Automatic Control, vol. 43, no. 12, pp. 1739-1745, 1998.

[42] Z. Dachang, D. Baolin, Z. Puchen, and W. Wu, "Adaptive backstepping sliding mode control of trajectory tracking for robotic manipulators," Complexity, vol. 2020, Article ID 3156787, 11 pages, 2020.

[43] C. Safa, E. Najib, and F. M'Sahli, "Nonsingular terminal sliding mode adaptive fuzzy type 2 control based on extended state observer," in Proceedings of the 16th International MultiConference on Systems, Signals \& Devices (SSD), pp. 102-107, Istanbul, Turkey, March 2019.

[44] C. Safa, K. Marwen, and F. M'Sahli, "Fuzzy T2I adaptive backstepping control for a state-coupled two-tank system," in Proceedings of the 20th International Conference on Sciences and Techniques of Automatic Control and Computer Engineering (STA), pp. 7-12, Sfax, Tunisia, 2020. 